\title{
Convergence and quasi-optimality of an adaptive finite element method for controlling $L_{2}$ errors
}

\author{
Alan Demlow • Rob Stevenson
}

Received: 9 November 2009 / Revised: 5 August 2010 / Published online: 15 December 2010

C The Author(s) 2010. This article is published with open access at Springerlink.com

\begin{abstract}
In this paper, a contraction property is proved for an adaptive finite element method for controlling the global $L_{2}$ error on convex polyhedral domains. Furthermore, it is shown that the method converges in $L_{2}$ with the best possible rate. The method that is analyzed is the standard adaptive method except that, if necessary, additional refinements are made to keep the meshes sufficiently mildly graded. This modification does not compromise the quasi-optimality of the resulting algorithm.
\end{abstract}

Mathematics Subject Classification (2000) $\quad 65$ N15 $\cdot 65$ N30

\section{Introduction and results}

Consider the elliptic model problem

$$
\begin{aligned}
-\Delta u & =f \text { in } \Omega, \\
u=0 & \text { on } \partial \Omega,
\end{aligned}
$$

A. Demlow was partially supported by National Science Foundation Grant DMS-0713770.

\footnotetext{
A. Demlow $(\varangle)$

Department of Mathematics, University of Kentucky,

715 Patterson Office Tower, Lexington, KY 40506-0027, USA

e-mail: demlow@ms.uky.edu

R. Stevenson

Korteweg-de Vries (KdV) Institute for Mathematics, University of Amsterdam, P.O. Box 94248, 1090 GE Amsterdam, The Netherlands

e-mail: R.P.Stevenson@uva.nl
} 
where $\Omega \subset \mathbb{R}^{n}, n \geq 2$, is a convex polyhedral domain and $f \in L_{2}(\Omega)$. Convexity is imposed so that the (dual) problem is $H^{2}(\Omega)$ regular, which is used to show reliability of the residual based a posteriori $L_{2}(\Omega)$ error estimator. The results presented here can be generalized to other linear second order elliptic operators having sufficiently smooth coefficients, but we present the simplest case in order to focus on essential ideas.

In this work we prove contraction and quasi-optimality properties for an adaptive finite element method (AFEM) for controlling errors in the $L_{2}(\Omega)$-norm. An AFEM is an iterative feedback procedure of the form

$$
\text { solve } \rightarrow \text { estimate } \rightarrow \text { mark } \rightarrow \text { refine }
$$

Such adaptive algorithms have for many years been a standard tool for efficiently approximating solutions to partial differential equations such as (1.1). The convergence properties of AFEM have become the subject of intense theoretical study only in the past few years, however. We refer to [6,11-14] for an overview of progress in basic convergence theory for AFEM for linear elliptic problems. Optimal convergence rates were demonstrated in $[4,19]$. A common feature of most theoretical results published to date concerning convergence and quasi-optimality of AFEM is that the error estimated in the "estimate" step in (1.2) is the global energy error. In particular, AFEM optimality results for error notions whose analysis require non-trivial duality arguments have not to our knowledge appeared in the literature. AFEM for controlling other norms are sometimes of practical interest, however; in the current context we refer for example to $[5,9,24]$ where "pollution effects" of global solution properties on the local energy error are measured and controlled adaptively in $L_{2}$.

In order to describe our results, let $\left(\mathcal{T}_{i}\right)_{i \geq 0}$ be a nested sequence of conforming, uniformly shape regular partitions of $\Omega$ produced by the AFEM. In this work, we consider simplicial partitions as they are produced by the newest vertex bisection algorithm or by its generalization to more than two dimensions. Let $S_{i} \subset H_{0}^{1}(\Omega)$ be the standard Lagrange finite element space of some fixed degree $k$ on $\mathcal{T}_{i}$, and let $u_{i} \in S_{i}$ satisfy

$$
A\left(u_{i}, v_{i}\right):=\int_{\Omega} \nabla u_{i} \cdot \nabla v_{i} \mathrm{~d} x=\int_{\Omega} f v_{i} \mathrm{~d} x, \quad\left(v_{i} \in S_{i}\right) .
$$

We also let $\||v|\|:=\sqrt{A(v, v)}$ and $\|\mid v\|_{D}:=\left(\int_{D}|\nabla v|^{2} \mathrm{~d} x\right)^{1 / 2}$ denote the global and local energy (semi)norms over $D$, respectively.

Our first goal in this work is to prove that the AFEM for controlling the $L_{2}$-error $\left\|u-u_{i}\right\|_{L_{2}(\Omega)}$ is majorized linearly convergent. In order to obtain this result, we require that the sequence $\left(\mathcal{T}_{i}\right)_{i}$ of partitions is sufficiently mildly graded. We will modify the "refine" routine so that, if necessary, it bisects additional simplices in order to preserve the mild grading of the partition. With this modification, and under the assumption that $\Omega$ is convex, in a sequence of estimates we prove that the quantities $\left\|u-u_{i}\right\|_{L_{2}(\Omega)}^{2}+\operatorname{osc}_{i}^{2},\left\|h_{i}\left(u-u_{i}\right)\right\|^{2}+\operatorname{osc}_{i}^{2}$ and $\eta_{i}^{2}$ are all uniformly equivalent. Here $\operatorname{osc}_{i}$ is the $L_{2}$-oscillation term, $h_{i} \in W_{\infty}^{1}(\Omega)$ is a regularized local mesh size function, and $\eta_{i}$ is the residual based $L_{2}$ a posteriori error estimator. A key ingredient 
of many of our proofs is that thanks to the mild grading of the partitions, the mesh size function can be designed so that $\left\|\nabla h_{i}\right\|_{L_{\infty}(\Omega)}$ is sufficiently small. Following an idea from [4], we then show that a suitably weighted sum of $\left\|\mid h_{i}\left(u-u_{i}\right)\right\| \|^{2}$ and $\eta_{i}^{2}$ is contracted by the AFEM, which implies the convergence result.

The approach of treating the $L_{2}$-error as a weighted energy error and then employing techniques developed for proving convergence of AFEM for global energy errors was used earlier in [5] to prove convergence of an AFEM for controlling local energy errors. We note that the restriction that $\left\|\nabla h_{i}\right\|_{L_{\infty}(\Omega)}$ must be sufficiently small has previously appeared in the literature in connection with a priori and a posteriori estimates in $L_{\infty}$ (cf. [7,8]) and a priori estimates in $L_{2}$ (cf. [3]).

Convergence of AFEM for controlling $L_{2}$-errors was also addressed in [13]. There are several substantial differences between our approach and the one taken in the latter work. Morin et al. [13] obtains convergence of AFEM for controlling weak norms under quite general assumptions on the marking strategy and norm of interest. While the assumptions are general, the convergence result obtained is correspondingly weak in that no estimate of the rate of convergence is obtained. In contrast, we require stronger assumptions; aside from the above-mentioned mesh restriction we also require a Dörfler-type marking strategy. The result is a much stronger convergence theory.

The second main theorem of this work states that for the sequence of partitions produced by the AFEM, $\left\|u-u_{i}\right\|_{L_{2}(\Omega)}+\operatorname{osc}_{i}$ can be bounded by some absolute multiple of $\left(\# \mathcal{T}_{i}\right)^{-s}$ for the best possible value of $s$. To arrive at this quasi-optimality result, we prove a localized a posteriori upper bound for the $L_{2}$-difference between Galerkin solutions on nested partitions. Furthermore, we show that, up to an oscillation term, the Galerkin solution is a near best approximation to $u$ from the finite element space in the $L_{2}$-norm. The latter result is of some interest independent of the current context, since it is known that the Ritz projection is not stable in the $L_{2}$ norm (cf. [1]). Both of these results rely on the condition that $\left\|\nabla h_{i}\right\|_{L_{\infty}(\Omega)}$ is sufficiently small. Finally, we show that, even if additional bisections are made to preserve the mild grading of the partitions, at any stage in the AFEM the cardinality of the current partition can be bounded by the cardinality of the initial partition plus some absolute multiple of the number of all elements that so far were marked for refinement by the routine "mark".

We finally comment on the practicality of the AFEM for which we prove optimality. Existing AFEM optimality results for global energy norms require that an essential user-supplied parameter in the "mark" step be sufficiently small. The $L_{2}$ AFEM for which we prove optimality requires two user-supplied parameters to be sufficiently small, one in the "mark" and the other in the "refine" step. Whereas in the energy case an upper bound for the parameter in terms of interpolation (Poincaré) constants can in principal be derived theoretically, this will be harder in the $L_{2}$-case as the corresponding parameters additionally depend on $H^{2}$ regularity constants.

It should also be noted that enforcing mild mesh grading may exacerbate the asymptotic nature of our results, since meshes satisfying a mild grading assumption with small $\mu$ will essentially remain quasi-uniform over the first iterations of the adaptive algorithm. On the other hand, practical experience seems to indicate that optimality in $L_{2}$ is obtained without taking precautions to keep the mesh sufficiently mildly graded, although there is no proof of that. This might mean either that that mildly gradedness is not really needed, or that that the typical meshes resulting from the application of an 
adaptive routine are automatically sufficiently mildly graded. Concerning the latter, note that a mesh that is optimal for controlling $L_{2}$ errors is more mildly graded than that for $H^{1}$ errors.

The paper is organized as follows. Sections 2 and 3 contain a number of preliminaries and definitions. In Sect. 4 we establish relationships between the error notions used in the paper. Our $L_{2}$ AFEM is defined precisely in Sect. 5. Sections 6 and 7 contain proofs of convergence and optimality of our AFEM, respectively. Section 8 contains discussion of the relationship between optimality of AFEM for controlling energy and $L_{2}$ norms and a numerical example. Finally, a bisection routine which preserves a mild mesh grading is given in Appendix A along with results concerning existence and properties of mesh functions.

\section{Preliminaries}

\subsection{Partitions and mesh functions}

Let $\mathcal{T}_{0}$ be a conforming partition of $\bar{\Omega}$ into (essentially) disjoint closed $n$-simplices. By fixing a local numbering of all vertices of all $T \in \mathcal{T}_{0}$, all possible descendants $\mathcal{T}$ of $\mathcal{T}_{0}$ that can be created by newest vertex bisection are uniquely determined. Here, with newest vertex bisection, we mean either the refinement procedure as it was developed in two space dimensions, or its generalization to any space dimension. Details can be found in Appendix A. The simplices in any of those partitions are uniformly shape regular, dependent only on the shape regularity parameters of $\mathcal{T}_{0}$ and the dimension $n$.

Generally, a descendant of $\mathcal{T}_{0}$ is non-conforming. Yet, with a suitable numbering of the vertices in the initial partition, any descendant can be refined to a conforming partition by inflating the total number of simplices by not more than some absolute multiple. Possibly after an initial refinement of the original initial partition, such a numbering always exists. Assuming such a numbering, we denote the set of all conforming descendants $\mathcal{T}$ of $\mathcal{T}_{0}$ by $\mathbb{T}$. For $\mathcal{T}, \tilde{\mathcal{T}} \in \mathbb{T}$, we will write $\mathcal{T} \subset \tilde{\mathcal{T}}$ when $\tilde{\mathcal{T}}$ is a refinement of $\mathcal{T}$ (or is equal to $\mathcal{T}$ ).

For $T \in \mathcal{T} \in \mathbb{T}$, let $h_{T}=|T|^{1 / n}$. Furthermore, let $\omega_{T}, \tilde{\omega}_{T}$ be the patches of elements (in $\mathcal{T}$ ) sharing a vertex or a facet (edge, face, etc.) with $T$. For $\mathcal{T} \in \mathbb{T}$, we construct the continuous piecewise linear "mesh-function" $h_{\mathcal{T}}$ by defining, for any vertex $z$ of $\mathcal{T}, h_{\mathcal{T}}(z)$ as the average of the $h_{T^{\prime}}$ over all $T^{\prime} \in \mathcal{T}$ with $T^{\prime} \ni z$. For some constants $c_{\mathbb{T}}$ and $C_{\mathbb{T}}$, it satisfies

$$
c_{\mathbb{T}} h_{T} \leq\left. h_{\mathcal{T}}\right|_{T} \leq C_{\mathbb{T}} h_{T} \quad(T \in \mathcal{T}, \mathcal{T} \in \mathbb{T})
$$

By the uniform shape regularity, for another constant $\hat{C}_{\mathbb{T}}$ we have

$$
\left\|\nabla h_{\mathcal{T}}\right\|_{L_{\infty}(\Omega)} \leq \hat{C}_{\mathbb{T}}(\mathcal{T} \in \mathbb{T})
$$


For a number of our results, we will need a mesh function $h_{\mathcal{T}}$ that for some sufficiently small, but fixed constant $\mu>0$ satisfies both

$$
\left\|\nabla h_{\mathcal{T}}\right\|_{L_{\infty}(\Omega)} \leq \mu,
$$

and (2.1) for some absolute constants $c_{\mathbb{T}}$ and $C_{\mathbb{T}}$ that are independent of $\mu$. By an application of the mean value theorem, the existence of such a mesh function $h_{\mathcal{T}}$ implies that $h_{T} \leq c_{\mathbb{T}}^{-1}\left(C_{\mathbb{T}} h_{T^{\prime}}+\mu \operatorname{dist}\left(T, T^{\prime}\right)\right)\left(T, T^{\prime} \in \mathcal{T}\right)$, and so in particular that $\max _{T \in \mathcal{T}} h_{T} \leq c_{\mathbb{T}}^{-1}\left(C_{\mathbb{T}} \min _{T \in \mathcal{T}} h_{T}+\mu \operatorname{diam} \Omega\right)$. We conclude that the existence of such an $h_{\mathcal{T}}$ imposes a restriction on the grading of the partition $\mathcal{T}$ beyond that imposed by shape regularity alone.

In Appendix A, we show that given a parameter $\mu$, there is a class of sufficiently mildly graded partitions $\mathcal{T} \in \mathbb{T}$ such that mesh functions $h_{\mathcal{T}}$ satisfying (2.1) independent of $\mu$ and (2.2) can be constructed. Given a $\mu>0$, we denote the class of such partitions by $\mathbb{T}_{\mu}$. The initial partition $\mathcal{T}_{0}$, as any of its uniform refinements, has no grading and is therefore contained in $\mathbb{T}_{\mu}$ for any $\mu$. Each time that we apply a mesh function $h_{\mathcal{T}}$ for a $\mathcal{T} \in \mathbb{T}_{\mu}$, obviously we mean the mesh function that satisfies (2.1) and (2.2), independent of $\mu$. These mesh functions are constructed such that, additionally, they are pointwise non-increasing under refinements inside the class $\mathbb{T}_{\mu}$. Finally, it is shown that any $\mathcal{T} \in \mathbb{T}$ can be refined to a partition in $\mathbb{T}_{\mu}$ by inflating the number of simplices by not more than some fixed multiple, dependent on $\mu$.

The restriction that $\left\|\nabla h_{\mathcal{T}}\right\|_{L_{\infty}(\Omega)}$ is sufficiently small appeared previously in [7], where a priori $L_{\infty}$-estimates were proved under this assumption. There $\mu$ was allowed to depend logarithmically on $\min _{T \in \mathcal{T}_{i}} h_{T}$. In the introductory Chapter 0 of the standard text [3], a priori $L_{2}$-estimates are also proved under the assumption (2.2) in the one-dimensional case; higher-dimensional versions of this result require a slightly more involved technical development but may be obtained with a similar proof. To our knowledge, (2.2) is the least restrictive assumption under which optimal a priori bounds in the $L_{2}$ norm have appeared in the literature for general space dimension $n$.

Finally, it is pointed out in [7] that the graded meshes typically needed to resolve corner singularities occurring in elliptic problems on corner domains satisfy (2.2) with $\mu$ small. Thus although we modify the standard "refine" routine so that it bisects additional simplices in order to preserve the mild grading of the partition if necessary, it may be that as in our numerical example in Sect. 8 additional bisections never have to be made.

\subsection{Finite element spaces and interpolants}

Given $\mathcal{T} \in \mathbb{T}$, let $S_{\mathcal{T}} \subset H_{0}^{1}(\Omega)$ be a space of continuous Lagrange finite element functions which are piecewise polynomials of some fixed degree $k$ on $\mathcal{T}$. As $u$ always denotes the solution of the continuous problem (1.1), the notation $u_{\mathcal{T}}$ will be reserved for the solution of its Galerkin discretization $A\left(u_{\mathcal{T}}, v_{\mathcal{T}}\right)=\int_{\Omega} f v_{\mathcal{T}} d x\left(v_{\mathcal{T}} \in S_{\mathcal{T}}\right)$. Note that $S_{\mathcal{T}} \subset S_{\tilde{\mathcal{T}}}$ whenever $\mathcal{T} \subset \tilde{\mathcal{T}}$.

We will employ two standard finite element (quasi)-interpolators onto $S_{\mathcal{T}}$. The first is the Lagrange interpolator, which we denote by $I_{L, \mathcal{T}}$. We will also employ 
the Scott-Zhang interpolator $I_{S Z, \mathcal{T}}$, which is stable in $H^{1}(\Omega)$, uniformly in $\mathcal{T} \in \mathbb{T}$ (cf. [18]). We do not list further properties of $I_{S Z, \mathcal{T}}$ here, as its application in the establishment of residual-type a posteriori upper bounds is now rather standard.

\subsection{Regularity}

Using that $f \in L_{2}(\Omega)$ and $\Omega$ is convex, the following $H^{2}$-regularity result is well-known.

Theorem 1 The solution $u$ of (1.1) satisfies $u \in H^{2}(\Omega)$ with

$$
\|u\|_{H^{2}(\Omega)} \leq C_{\text {reg }}\|f\|_{L_{2}(\Omega)} .
$$

\subsection{Constants}

In what follows, we shall denote by $C$ and $C_{i}(i=1,2,3 \ldots)$ generic constants that may depend on the shape regularity parameters of $\mathcal{T}_{0}$, the space dimension $n$, the polynomial degree $k$, the constants $c_{\mathbb{T}}$ and $C_{\mathbb{T}}$ in (2.2), and the $H^{2}$-regularity constant $C_{\text {reg }}$ from the theorem above. Other constants will be defined as necessary. In addition, we will often write $a \lesssim b$ instead of $a \leq C b$ with $C$ as above. Obviously, with $a \gtrsim b$ it is meant that $b \lesssim a$, and with $a \approx b$ that both $a \lesssim b$ and $a \gtrsim b$.

\section{Residual based $L_{2}$ a posteriori error estimator}

Given $\mathcal{T} \in \mathbb{T}, v_{\mathcal{T}} \in S_{\mathcal{T}}$, and $T \in \mathcal{T}$, we define the $L_{2}$-type error indicator $\eta\left(v_{\mathcal{T}}, T\right)$ by

$$
\eta\left(v_{\mathcal{T}}, T\right)^{2}=h_{T}^{4}\left\|f+\Delta v_{\mathcal{T}}\right\|_{L_{2}(T)}^{2}+h_{T}^{3}\left\|\llbracket \nabla v_{\mathcal{T}} \rrbracket\right\|_{L_{2}(\partial T \backslash \partial \Omega)}^{2} .
$$

We omit $v_{\mathcal{T}}$ in the above notation when $v_{\mathcal{T}}=u_{\mathcal{T}}$, that is, $\eta(T)=\eta\left(u_{\mathcal{T}}, T\right)$. Next we define error estimators. If $\mathcal{M} \subset \mathcal{T}$, we define

$$
\eta\left(v_{\mathcal{T}}, \mathcal{M}\right)^{2}=\sum_{T \in \mathcal{M}} \eta\left(v_{\mathcal{T}}, T\right)^{2},
$$

If $v_{\mathcal{T}}=u_{\mathcal{T}}$, we shall omit the reference to $v_{\mathcal{T}}$ in our notation as above.

The following two results dealing with the reliability and efficiency of the estimator are well-known. Proofs can be found in [22]. The first result makes use of the fact that $\Omega$ is convex.

Proposition 1 It holds that

$$
\left\|u-u_{\mathcal{T}}\right\|_{L_{2}(\Omega)} \lesssim \eta(\mathcal{T}) \quad(\mathcal{T} \in \mathbb{T})
$$

Proposition 2 There exists a constant $C_{1}>0$ such that

$$
\eta\left(v_{\mathcal{T}}, \mathcal{T}\right) \leq C_{1}\left[\left\|u-v_{\mathcal{T}}\right\|_{L_{2}(\Omega)}+\operatorname{osc}(\mathcal{T})\right] \quad\left(\mathcal{T} \in \mathbb{T}, v_{\mathcal{T}} \in S_{\mathcal{T}}\right)
$$


Here $\operatorname{osc}(\mathcal{M})(\mathcal{M} \subset \mathcal{T})$, known as the data oscillation term, is defined by

$$
\operatorname{osc}(\mathcal{M})^{2}=\sum_{T \in \mathcal{M}} h_{T}^{4}\left\|f-f_{T}\right\|_{L_{2}(T)}^{2}
$$

where $f_{T}$ is the $L_{2}(T)$-projection of $\left.f\right|_{T}$ onto $\mathbb{P}_{k-1}(T)$.

Remark 1 Note that $\operatorname{osc}(\mathcal{M}) \leq \eta\left(v_{\mathcal{T}}, \mathcal{M}\right)\left(\mathcal{M} \subset \mathcal{T} \in \mathbb{T}, v_{\mathcal{T}} \in S_{\mathcal{T}}\right)$.

Remark 2 Efficient a posteriori control of the $L_{2}$ error when $H^{2}$ regularity is not present is significantly more difficult than in the present case. When $n=2$, reliable estimators which control $\left\|u-u_{i}\right\|_{L_{2}(\Omega)}$ on non-convex polygonal domains by explicitly taking corner singularities into account can be found in [9]; these estimators do not however satisfy a corresponding a posteriori lower bound. Reliable and efficient estimators for bounding a weighted $L_{2}$ norm on non-convex polygonal domains can be found in [23]. Extension of both of these results to $n=3$ is technically difficult because they rely on knowledge of the exact nature of corner singularities. Analysis of AFEM based on these estimators also would present technical difficulties beyond those encountered in our analysis here for the most basic case, so we restrict our attention to situations with $H^{2}$ regularity.

\section{Relations between several error notions}

In this section we shall establish several relations between different error notions that will be used in the succeeding sections to prove convergence and quasi-optimality of AFEM in the $L_{2}(\Omega)$-norm.

Instead of directly proving that an AFEM for controlling $\left\|u-u_{i}\right\|_{L_{2}(\Omega)}$ is contractive, we shall show that a total error notion based on ||$h_{i}\left(u-u_{i}\right)|| \mid$ contracts. The following proposition establishes that this error notion is meaningful so long as the mesh grading is sufficiently mild. It will be used that $\Omega$ is convex.

Proposition 3 For sufficiently small $\mu$, let $\mathcal{T} \in \mathbb{T}_{\mu}$. Then

$$
\left\|u-u_{\mathcal{T}}\right\|_{L_{2}(\Omega)} \lesssim\left\|h_{\mathcal{T}}\left(u-u_{\mathcal{T}}\right)\right\|
$$

Proof We first employ a duality argument. Let $e=u-u_{\mathcal{T}}$, and let $z \in H^{2}(\Omega) \cap$ $H_{0}^{1}(\Omega)$ satisfy $A(v, z)=(v, e)$ for all $v \in H_{0}^{1}(\Omega)$. Employing Galerkin orthogonality, approximation properties, Theorem $1,\left.h_{\mathcal{T}}\right|_{T} \approx h_{T}$, and $\left\|\nabla h_{\mathcal{T}}\right\|_{L_{\infty}(\Omega)} \leq \mu$, we have that 


$$
\begin{aligned}
(e, e) & =A(e, z) \\
& =A\left(e, z-I_{S Z, \mathcal{T} z} \lesssim\left\|h_{\mathcal{T}} \nabla e\right\|_{L_{2}(\Omega)}\left\|h_{\mathcal{T}}^{-1} \nabla\left(z-I_{S Z, \mathcal{T}} z\right)\right\|_{L_{2}(\Omega)}\right. \\
& \lesssim\left(\left\|h_{\mathcal{T}} e\right\|\|+\| e \nabla h_{\mathcal{T}} \|_{L_{2}(\Omega)}\right)\|z\|_{H^{2}(\Omega)} \\
& \lesssim\|\| h_{\mathcal{T}} e\|\| e\left\|_{L_{2}(\Omega)}+\mu\right\| e \|_{L_{2}(\Omega)}^{2} .
\end{aligned}
$$

Taking $\mu$ sufficiently small to kick back the last term completes the proof.

Next we prove that the a posteriori error estimator provides also an upper bound for $\left\|h_{\mathcal{T}}\left(u-u_{\mathcal{T}}\right)\right\| \|$. Again, it is used that $\Omega$ is convex.

Proposition 4 For any $\mathcal{T} \in \mathbb{T}$,

$$
\left\|\left|h_{\mathcal{T}}\left(u-u_{\mathcal{T}}\right) \|\right| \lesssim \eta(\mathcal{T})\right.
$$

Proof Letting $e=u-u_{\mathcal{T}}$, after a short computation and inserting Proposition 1 while recalling that $\left\|\nabla h_{\mathcal{T}}\right\|_{L_{\infty}(\Omega)} \lesssim 1$, we find that

$$
\begin{aligned}
A\left(h_{\mathcal{T}} e, h_{\mathcal{T}} e\right) & =A\left(e, h_{\mathcal{T}}^{2} e\right)+\left\|e \nabla h_{\mathcal{T}}\right\|_{L_{2}(\Omega)}^{2} \\
& \leq\left|A\left(e, h_{\mathcal{T}}^{2} e\right)\right|+\left\|\nabla h_{\mathcal{T}}\right\|_{L_{\infty}(\Omega)}^{2}\|e\|_{L_{2}(\Omega)}^{2} \\
& \lesssim\left|A\left(e, h_{\mathcal{T}}^{2} e\right)\right|+\eta(\mathcal{T})^{2} .
\end{aligned}
$$

Using standard techniques for proving residual-type energy estimates along while recalling that $\left.h_{\mathcal{T}}\right|_{T} \approx h_{T}$ and $\left\|\nabla h_{\mathcal{T}}\right\|_{L_{\infty}(\Omega)} \lesssim 1$ yields

$$
\begin{aligned}
A\left(e, h_{\mathcal{T}}^{2} e\right)= & A\left(e, h_{\mathcal{T}}^{2} e-I_{S Z, \mathcal{T}}\left(h_{\mathcal{T}}^{2} e\right)\right) \\
\lesssim & \sum_{T \in \mathcal{T}}\left(h_{T}\left\|\Delta u_{\mathcal{T}}+f\right\|_{L_{2}(T)}+h_{T}^{1 / 2}\left\|\llbracket \nabla u_{\mathcal{T}} \rrbracket\right\|_{L_{2}(\partial T)}\right) \\
& \times\left\|\nabla\left(h_{\mathcal{T}}^{2} e\right)\right\|_{L_{2}\left(\omega_{T}\right)} \\
\lesssim & \sum_{T \in \mathcal{T}} \eta(T)\left(\left\|\nabla\left(h_{\mathcal{T}} e\right)\right\|_{L_{2}\left(\omega_{T}\right)}+\left\|\nabla h_{\mathcal{T}}\right\|_{L_{\infty}\left(\omega_{T}\right)}\|e\|_{L_{2}\left(\omega_{T}\right)}\right) \\
\lesssim & \eta(\mathcal{T})\left(\left\|h_{\mathcal{T}} e\right\|+\|e\|_{L_{2}(\Omega)}\right)
\end{aligned}
$$

Inserting the result of Proposition 1 into (4.3), inserting the result into (4.2), and kicking back the term $\left\|\left|h_{\mathcal{T}} e \|\right|\right.$ above completes the proof.

Next we prove a stability result for the error indicator, cf. Proposition 3.3 of [4].

\section{Lemma 1 It holds that}

$$
|\eta(v, T)-\eta(w, T)| \lesssim||\left|h_{\mathcal{T}}(v-w)\right|||_{\tilde{\omega}_{T}}+\left\|\nabla h_{\mathcal{T}}\right\|_{L_{\infty}\left(\tilde{\omega}_{T}\right)}\|v-w\|_{L_{2}\left(\tilde{\omega}_{T}\right)}
$$

$\left(T \in \mathcal{T} \in \mathbb{T}, v, w \in S_{\mathcal{T}}\right)$. 
Proof Recalling the definition (3.1), we use the triangle inequality to compute for $T \in \mathcal{T}_{i}$ that

$$
\eta(v, T) \leq \eta(w, T)+\left(h_{T}^{4}\|\Delta(v-w)\|_{L_{2}(T)}^{2}+h_{T}^{3}\|\llbracket \nabla(v-w) \rrbracket\|_{L_{2}(\partial T)}^{2}\right)^{1 / 2} .
$$

Employing an inverse inequality and $\left.h_{\mathcal{T}}\right|_{T} \approx h_{T}$, we compute

$$
\begin{aligned}
h_{T}^{2}\|\Delta(v-w)\|_{L_{2}(T)} & \lesssim h_{T}\|\nabla(v-w)\|_{L_{2}(T)} \\
& \lesssim\left\|\nabla\left[h_{\mathcal{T}}(v-w)\right]\right\|_{L_{2}(T)}+\left\|(v-w) \nabla h_{\mathcal{T}}\right\|_{L_{2}(T)} .
\end{aligned}
$$

We next compute as above while employing the trace inequality $\|\nabla v\|_{L_{2}(\partial T)} \lesssim$ $h_{T}^{-1 / 2}\|\nabla v\|_{L_{2}(T)}+h_{T}^{1 / 2}|v|_{H^{2}(T)}$ to obtain for the edge $e=T \cap T^{\prime}$

$$
\begin{aligned}
& h_{T}^{3 / 2}\|\llbracket \nabla(v-w) \rrbracket\|_{L_{2}(e)} \\
& \leq h_{T}^{3 / 2}\left(\left\|\nabla(v-w)_{T}\right\|_{L_{2}(e)}+\left\|\nabla(v-w)_{T^{\prime}}\right\|_{L_{2}(e)}\right) \\
& \lesssim h_{T}^{3 / 2}\left(h_{T}^{-1 / 2}\|\nabla(v-w)\|_{L_{2}(T)}+h_{T}^{1 / 2}|v-w|_{H^{2}(T)}\right. \\
& \left.\quad+h_{T}^{-1 / 2}\|\nabla(v-w)\|_{L_{2}\left(T^{\prime}\right)}+h_{T}^{1 / 2}|v-w|_{H^{2}\left(T^{\prime}\right)}\right) \\
& \lesssim\|\| h_{\mathcal{T}}(v-w)\|\|_{T \cup T^{\prime}}+\left\|(v-w) \nabla h_{\mathcal{T}}\right\|_{L_{2}\left(T \cup T^{\prime}\right)} .
\end{aligned}
$$

Above we have employed the convention that for $x$ lying in the edge $e=T \cap T^{\prime}$, $\nabla(v-w)_{T}(x)=\lim _{y \in T, y \rightarrow x} \nabla(v-w)(y)$. Noting that $\left\|(v-w) \nabla h_{\mathcal{T}}\right\|_{L_{2}\left(T \cup T^{\prime}\right)} \leq$ $\left\|\nabla h_{\mathcal{T}}\right\|_{L_{\infty}\left(\tilde{\omega}_{T}\right)}\|v-w\|_{L_{2}\left(\tilde{\omega}_{T}\right)}$, summing over the edges of $T$, and collecting (4.5) and (4.6) into (4.4) completes the proof.

The following localized upper bound will be used for proving quasi-optimality of the AFEM. It will be used that $\Omega$ is convex.

Lemma 2 There exists a constant $C_{2}>0$ such that for sufficiently small $\mu, \mathcal{T} \in \mathbb{T}_{\mu}$, and $\mathcal{T} \subset \tilde{\mathcal{T}} \in \mathbb{T}$,

$$
\left\|u_{\tilde{\mathcal{T}}}-u_{\mathcal{T}}\right\|_{L_{2}(\Omega)} \leq C_{2} \eta\left(\mathcal{R}_{\mathcal{T} \rightarrow \tilde{\mathcal{T}}}\right)
$$

Here $\mathcal{R}_{\mathcal{T} \rightarrow \tilde{\mathcal{T}}} \subset \mathcal{T}$ is the subset of elements that are refined in passing from $\mathcal{T}$ to $\tilde{\mathcal{T}}$.

Proof We set $E=u_{\tilde{\mathcal{T}}}-u_{\mathcal{T}}$, so that $A(E, \chi)=0$ for all $\chi \in S_{\mathcal{T}}$. Arguing as in Proposition 3 , it is easy to show that

$$
\|E\|_{L_{2}(\Omega)} \lesssim\left\|h_{\mathcal{T}} \nabla E\right\|_{L_{2}(\Omega)}
$$

An elementary computation yields for $\epsilon>0$

$$
\begin{aligned}
\left\|h_{\mathcal{T}} \nabla E\right\|_{L_{2}(\Omega)}^{2} & =\left(h_{\mathcal{T}} \nabla E, h_{\mathcal{T}} \nabla E\right)=A\left(E, h_{\mathcal{T}}^{2} E\right)-2\left(h_{\mathcal{T}} \nabla E, E \nabla h_{\mathcal{T}}\right) \\
& \leq\left|A\left(E, h_{\mathcal{T}}^{2} E\right)\right|+\epsilon\left\|h_{\mathcal{T}} \nabla E\right\|_{L_{2}(\Omega)}^{2}+\frac{\mu^{2}}{\epsilon}\|E\|_{L_{2}(\Omega)}^{2} .
\end{aligned}
$$


Taking $\epsilon$ small enough to kick back the second term above and inserting the result into (4.7) yields

$$
\left\|h_{\mathcal{T}} \nabla E\right\|_{L_{2}(\Omega)}^{2} \lesssim\left|A\left(E, h_{\mathcal{T}}^{2} E\right)\right|+\mu^{2}\|E\|_{L_{2}(\Omega)},
$$

so that by (4.7) for $\mu$ sufficiently small we have

$$
\left\|h_{\mathcal{T}} \nabla E\right\|_{L_{2}(\Omega)}^{2} \lesssim\left|A\left(E, h_{\mathcal{T}}^{2} E\right)\right|
$$

Let $I_{L, \tilde{\mathcal{T}}}: C_{0}(\Omega) \rightarrow S_{\tilde{\mathcal{T}}}$ and $I_{S Z, \mathcal{T}}$ be as in Sect. 2.2. It is shown in [4, Lemma 3.6] that $I_{S Z, \mathcal{T}}$ may be defined so that for $T \in \mathcal{T} \cap \tilde{\mathcal{T}}$ (i.e., for $T \in \mathcal{T} \backslash \mathcal{R}_{\mathcal{T} \rightarrow \tilde{\mathcal{T}}}$ ), $\left(\chi-I_{S Z, \mathcal{T}} \chi\right)_{T}=0$ for $\chi \in S_{\tilde{\mathcal{T}}}$. We assume that $I_{S Z, \mathcal{T}}$ is so defined. Then

$$
\begin{aligned}
A\left(E, h_{\mathcal{T}}^{2} E\right) & =\underbrace{A\left(E, h_{\mathcal{T}}^{2} E-I_{L, \tilde{\mathcal{T}}}\left(h_{\mathcal{T}}^{2} E\right)\right)}_{\mathrm{I}} \\
& +\underbrace{A\left(E, I_{L, \tilde{\mathcal{T}}}\left(h_{\mathcal{T}}^{2} E\right)-I_{S Z, \mathcal{T}}\left(I_{L, \tilde{\mathcal{T}}}\left(h_{\mathcal{T}}^{2} E\right)\right)\right)}_{\mathrm{II}} .
\end{aligned}
$$

In order to bound the term "I" in (4.11), in essence we employ the classical super approximation tool introduced in [15]. Restricted to $T \in \tilde{\mathcal{T}}$, we have $h_{\mathcal{T}} \in \mathbb{P}_{1}$ and $E \in \mathbb{P}_{k}$, and so $\left|D^{2} h_{\mathcal{T}}^{2}\right| \lesssim\left|\nabla h_{\mathcal{T}}\right|^{2}, D^{j} h_{\mathcal{T}}=0$ for $j \geq 3$, and $D^{k+1} E=0$. Employing inverse inequalities and using that $\left.h_{\mathcal{T}}\right|_{T^{\prime}} \approx h_{T^{\prime}}\left(T^{\prime} \in \mathcal{T}\right)$ and $\left\|\nabla h_{\mathcal{T}}\right\|_{L_{\infty}(\Omega)} \lesssim 1$, we thus compute

$$
\begin{aligned}
\left\|\nabla\left(h_{\mathcal{T}}^{2} E-I_{L, \tilde{\mathcal{T}}}\left(h_{\mathcal{T}}^{2} E\right)\right)\right\|_{L_{2}(T) \lesssim} & h_{T}^{k}\left\|D^{k+1}\left(h_{\mathcal{T}}^{2} E\right)\right\|_{L_{2}(T)} \\
\lesssim & h_{T}^{k}\left[\left\|\nabla h_{\mathcal{T}}^{2}\right\|_{L_{\infty}(T)}\left\|D^{k} E\right\|_{L_{2}(T)}\right. \\
& \left.+\left\|D^{2} h_{\mathcal{T}}^{2}\right\|_{L_{\infty}(T)}\left\|D^{k-1} E\right\|_{L_{2}(T)}\right] \\
\lesssim & h_{T}^{k}\left[\left\|h_{\mathcal{T}}\right\|_{L_{\infty}(T)}\left\|\nabla h_{\mathcal{T}}\right\|_{L_{\infty}(T)}\left\|D^{k} E\right\|_{L_{2}(T)}\right. \\
& \left.+\left\|\nabla h_{\mathcal{T}}\right\|_{L_{\infty}(T)}^{2}\left\|D^{k-1} E\right\|_{L_{2}(T)}\right] \\
\lesssim & {\left[\left\|h_{\mathcal{T}}\right\|_{L_{\infty}(T)}\left\|\nabla h_{\mathcal{T}}\right\|_{L_{\infty}(T)}\right.} \\
& \left.+h_{T}\left\|\nabla h_{\mathcal{T}}\right\|_{L_{\infty}(T)}^{2}\right]\|E\|_{L_{2}(T)} \\
\lesssim & \left\|h_{\mathcal{T}}\right\|_{L_{\infty}(T)}\left\|\nabla h_{\mathcal{T}}\right\|_{L_{\infty}(T)}\|E\|_{L_{2}(T)}
\end{aligned}
$$

Using that $\left\|\nabla h_{\mathcal{T}}\right\|_{L_{\infty}(\Omega)} \leq \mu$, for any $\epsilon>0$ it thus holds that

$$
\begin{aligned}
\text { |"I"| } & \lesssim \sum_{T \in \tilde{\mathcal{T}}}\left\|h_{\mathcal{T}} \nabla E\right\|_{L_{2}(T)} \mu\|E\|_{L_{2}(T)} \\
& \lesssim\left\|h_{\mathcal{T}} \nabla E\right\|_{L_{2}(\Omega)} \mu\|E\|_{L_{2}(\Omega)} \\
& \leq \epsilon\left\|h_{\mathcal{T}} \nabla E\right\|_{L_{2}(\Omega)}^{2}+\frac{\mu^{2}}{4 \epsilon}\|E\|_{L_{2}(\Omega)}^{2}
\end{aligned}
$$


To bound the term "II", we first note that since $h_{\mathcal{T}}^{2} E$ is piecewise polynomial on $\tilde{\mathcal{T}}$, the stability bound $\left\|\nabla I_{L, \tilde{\mathcal{T}}}\left(h_{\mathcal{T}}^{2} E\right)\right\|_{L_{2}(T)} \lesssim\left\|\nabla\left(h_{\mathcal{T}}^{2} E\right)\right\|_{L_{2}(T)}$ holds. Recall that $I_{L, \tilde{\mathcal{T}}}\left(h_{\mathcal{T}}^{2} E\right)-I_{S Z, \mathcal{T}}\left(I_{L, \tilde{\mathcal{T}}}\left(h_{\mathcal{T}}^{2} E\right)\right)$ is in $S_{\tilde{\mathcal{T}}}$ and vanishes on all $T \in \mathcal{T} \backslash \mathcal{R}_{\mathcal{T} \rightarrow \tilde{\mathcal{T}}}$. We may then compute using Galerkin orthogonality and standard residual techniques that for $\epsilon>0$,

$$
\begin{aligned}
& \text { |“'II"| }=\mid A\left(u-u_{\mathcal{T}}, I_{L, \tilde{\mathcal{T}}}\left(h_{\mathcal{T}}^{2} E\right)-I_{S Z, \mathcal{T}}\left(I_{L, \tilde{\mathcal{T}}}\left(h_{\mathcal{T}}^{2} E\right)\right) \mid\right. \\
& \lesssim \sum_{T \in \mathcal{R}_{\mathcal{T} \rightarrow \tilde{\mathcal{T}}}}\left(h_{T}\left\|f+\Delta u_{\mathcal{T}}\right\|_{L_{2}(T)}+h_{T}^{1 / 2}\left\|\llbracket \nabla u_{\mathcal{T}} \rrbracket\right\|_{L_{2}(\partial T)}\right) \\
& \times\left\|\nabla I_{L, \tilde{\mathcal{T}}}\left(h_{\mathcal{T}}^{2} E\right)\right\|_{L_{2}\left(\omega_{T}\right)} \\
& \lesssim \sum_{T \in \mathcal{R}_{\mathcal{T} \rightarrow \tilde{\mathcal{T}}}} h_{T}^{-1} \eta(T)\left\|\nabla\left(h_{\mathcal{T}}^{2} E\right)\right\|_{L_{2}\left(\omega_{T}\right)} \\
& \lesssim \sum_{T \in \mathcal{R}_{\mathcal{T} \rightarrow \tilde{\mathcal{T}}}} h_{T}^{-1} \eta(T) \\
& \times\left(h_{T}\left\|h_{\mathcal{T}} \nabla E\right\|_{L_{2}\left(\omega_{T}\right)}+h_{T} \mu\|E\|_{L_{2}\left(\omega_{T}\right)}\right) \\
& \lesssim \sum_{T \in \mathcal{R}_{\mathcal{T} \rightarrow \tilde{\mathcal{T}}}} \eta(T)\left(\left\|h_{\mathcal{T}} \nabla E\right\|_{L_{2}\left(\omega_{T}\right)}+\mu\|E\|_{L_{2}\left(\omega_{T}\right)}\right) \\
& \lesssim\left(1+\frac{1}{\epsilon}\right) \eta\left(\mathcal{R}_{\mathcal{T} \rightarrow \tilde{\mathcal{T}}}\right)^{2}+\epsilon\left\|h_{\mathcal{T}} \nabla E\right\|_{L_{2}(\Omega)}^{2}+\mu^{2}\|E\|_{L_{2}(\Omega)}^{2} .
\end{aligned}
$$

Inserting (4.13) and (4.14) into (4.11) yields for $\epsilon>0$,

$$
\begin{aligned}
\left\|h_{\mathcal{T}} \nabla E\right\|_{L_{2}(\Omega)}^{2} \lesssim & \left(1+\frac{1}{\epsilon}\right) \eta\left(\mathcal{R}_{\mathcal{T} \rightarrow \tilde{\mathcal{T}}}\right)^{2}+\epsilon\left\|h_{\mathcal{T}} \nabla E\right\|_{L_{2}(\Omega)}^{2} \\
& +\frac{\mu^{2}}{\epsilon}\|E\|_{L_{2}(\Omega)}^{2} .
\end{aligned}
$$

Taking $\epsilon$ small enough to kick back the second term above and then inserting the result into (4.10) yields

$$
\left\|h_{\mathcal{T}} \nabla E\right\|_{L_{2}(\Omega)}^{2} \lesssim \eta\left(\mathcal{R}_{\mathcal{T} \rightarrow \tilde{\mathcal{T}}}\right)^{2}+\mu^{2}\left\|h_{\mathcal{T}} \nabla E\right\|_{L_{2}(\Omega)}^{2}
$$

by (4.7). Taking $\mu$ small enough to kick back the last term above and again applying (4.7) completes the proof of the lemma.

We next prove a quasi-orthogonality result (cf. [11] for a similar estimate in the context of convergence of AFEM in the global energy norm for general second-order linear elliptic problems).

Lemma 3 For any $\epsilon>0, \mathcal{T} \in \mathbb{T}$, and $v_{\mathcal{T}} \in S_{\mathcal{T}}$, it holds that

$$
\begin{aligned}
& \left.\|\| h_{\mathcal{T}}\left(u-u_{\mathcal{T}}\right)\left\|^{2}+\right\|\left\|h_{\mathcal{T}}\left(u_{\mathcal{T}}-v_{\mathcal{T}}\right)\right\|\right|^{2}-(1+\epsilon)\left\|\mid h_{\mathcal{T}}\left(u-v_{\mathcal{T}}\right)\right\| \|^{2} \\
& \quad \lesssim \epsilon^{-1}\left\|\nabla h_{\mathcal{T}}\right\|_{L_{\infty}(\Omega)}^{2}\left(\left\|u-u_{\mathcal{T}}\right\|_{L_{2}(\Omega)}^{2}+\left\|u-v_{\mathcal{T}}\right\|_{L_{2}(\Omega)}^{2}\right) .
\end{aligned}
$$


Proof Writing $\tilde{e}_{\mathcal{T}}=u-v_{\mathcal{T}}$ and $e_{\mathcal{T}}=u-u_{\mathcal{T}}$, we calculate

$$
\begin{aligned}
\left\|h_{\mathcal{T}} e_{\mathcal{T}}\right\| \|^{2}= & \left\|h_{\mathcal{T}} \tilde{e}_{\mathcal{T}}\right\|\left\|^{2}-\right\| \mid h_{\mathcal{T}}\left(u_{\mathcal{T}}-v_{\mathcal{T}}\right)\|\|^{2} \\
& -2 A_{(}\left(h_{\mathcal{T}} e_{\mathcal{T}}, h_{\mathcal{T}}\left(u_{\mathcal{T}}-v_{\mathcal{T}}\right)\right) .
\end{aligned}
$$

An elementary calculation yields

$$
\begin{aligned}
& \left|A\left(h_{\mathcal{T}} e_{\mathcal{T}}, h_{\mathcal{T}}\left(u_{\mathcal{T}}-v_{\mathcal{T}}\right)\right)\right|=\mid A\left(e_{\mathcal{T}}, h_{\mathcal{T}}^{2}\left(u_{\mathcal{T}}-v_{\mathcal{T}}\right)\right) \\
& \quad+\left(\left|\nabla h_{\mathcal{T}}\right|^{2} e_{\mathcal{T}}, u_{\mathcal{T}}-v_{\mathcal{T}}\right)-\left(\tilde{e}_{\mathcal{T}} \nabla h_{\mathcal{T}}, \nabla\left(h_{\mathcal{T}} e_{\mathcal{T}}\right)\right) \\
& \quad+\left(e_{\mathcal{T}} \nabla h_{\mathcal{T}}, \nabla\left(h_{\mathcal{T}} \tilde{e}_{\mathcal{T}}\right)\right) \mid \\
& \leq\left\|\tilde{e}_{\mathcal{T}} \nabla h_{\mathcal{T}}\right\|_{L_{2}(\Omega)}\left|\left\|h _ { \mathcal { T } } e _ { \mathcal { T } } \left|\| + \| e _ { \mathcal { T } } \nabla h _ { \mathcal { T } } \left\|_ { L _ { 2 } ( \Omega ) } \left|\left\|h_{\mathcal{T}} \tilde{e}_{\mathcal{T}} \mid\right\|\right.\right.\right.\right.\right. \\
& \quad+\left\|e_{\mathcal{T}} \nabla h_{\mathcal{T}}\right\|_{L_{2}(\Omega)}\left\|\left(u_{\mathcal{T}}-v_{\mathcal{T}}\right) \nabla h_{\mathcal{T}}\right\|_{L_{2}(\Omega)} \\
& \quad+\left|A\left(e_{\mathcal{T}}, h_{\mathcal{T}}^{2}\left(u_{\mathcal{T}}-v_{\mathcal{T}}\right)\right)\right| .
\end{aligned}
$$

Arguments analogous to those in (4.12) and (4.13) yield

$$
\begin{aligned}
\left|A\left(e_{\mathcal{T}}, h_{\mathcal{T}}^{2}\left(u_{\mathcal{T}}-v_{\mathcal{T}}\right)\right)\right|= & \left|A\left(e_{\mathcal{T}},\left(I-I_{L, \mathcal{T}}\right) h_{\mathcal{T}}^{2}\left(u_{\mathcal{T}}-v_{\mathcal{T}}\right)\right)\right| \\
\lesssim & \left\|h_{\mathcal{T}} \nabla e_{\mathcal{T}}\right\|_{L_{2}(\Omega)}\left\|\nabla h_{\mathcal{T}}\right\|_{L_{\infty}(\Omega)}\left\|u_{\mathcal{T}}-v_{\mathcal{T}}\right\|_{L_{2}(\Omega)} \\
\leq & \left(\left\|h_{\mathcal{T}} e_{\mathcal{T}}\right\|+\left\|\nabla h_{\mathcal{T}}\right\|_{L_{\infty}(\Omega)}\left\|e_{\mathcal{T}}\right\|_{L_{2}(\Omega)}\right) \\
& \times\left\|\nabla h_{\mathcal{T}}\right\|_{L_{\infty}(\Omega)}\left\|u_{\mathcal{T}}-v_{\mathcal{T}}\right\|_{L_{2}(\Omega)} .
\end{aligned}
$$

Inserting this bound into (4.19) and applying Young's inequality a few times yields for some constant $C>0$ and any $\delta>0$ that

$$
\begin{aligned}
\left|A\left(h_{\mathcal{T}} e_{\mathcal{T}}, h_{\mathcal{T}}\left(u_{\mathcal{T}}-v_{\mathcal{T}}\right)\right)\right| \leq & \frac{\delta}{2}\left(\left\|\mid h_{\mathcal{T}} e_{\mathcal{T}}\right\|\left\|^{2}+\right\| h_{\mathcal{T}} \tilde{e}_{\mathcal{T}}\|\|^{2}\right) \\
& +C\left(1+\frac{1}{\delta}\right)\left\|\nabla h_{\mathcal{T}}\right\|_{L_{\infty}(\Omega)}^{2}\left(\left\|e_{\mathcal{T}}\right\|_{L_{2}(\Omega)}^{2}+\left\|\tilde{e}_{\mathcal{T}}\right\|_{L_{2}(\Omega)}^{2}\right) .
\end{aligned}
$$

Inserting (4.20) into (4.18) then yields

$$
\begin{aligned}
(1-\delta)\|\| h_{\mathcal{T}} e_{\mathcal{T}}\|\|^{2} \leq & (1+\delta)\left\|\mid h_{\mathcal{T}} \tilde{e}_{\mathcal{T}}\right\|\left\|^{2}-\right\| h_{\mathcal{T}}\left(u_{\mathcal{T}}-v_{\mathcal{T}}\right)\|\|^{2} \\
& +2 C\left(1+\frac{1}{\delta}\right)\left\|\nabla h_{\mathcal{T}}\right\|_{L_{\infty}(\Omega)}^{2}\left(\left\|e_{\mathcal{T}}\right\|_{L_{2}(\Omega)}^{2}+\left\|\tilde{e}_{\mathcal{T}}\right\|_{L_{2}(\Omega)}^{2}\right) .
\end{aligned}
$$

Dividing through by $1-\delta$ and selecting $\frac{1+\delta}{1-\delta}$ as $1+\epsilon$ completes the proof of Lemma 3 .

A combination of the Propositions 4 and 2 shows that

$$
\left\|\left|h_{\mathcal{T}}\left(u-u_{\mathcal{T}}\right)\|\mid \lesssim\| u-u_{\mathcal{T}} \|_{L_{2}(\Omega)}+\operatorname{osc}(\mathcal{T})\right.\right.
$$


In the following lemma it is shown that this inequality is even valid with $u_{\mathcal{T}}$ reading as any $v_{\mathcal{T}} \in S_{\mathcal{T}}$.

Lemma 4 For $\mathcal{T} \in \mathbb{T}$, it holds that

$$
\left\|\left|h_{\mathcal{T}}\left(u-v_{\mathcal{T}}\right)\|\mid \lesssim\| u-v_{\mathcal{T}} \|_{L_{2}(\Omega)}+\operatorname{osc}(\mathcal{T}) \quad\left(v_{\mathcal{T}} \in S_{\mathcal{T}}\right)\right.\right.
$$

Proof Writing $z_{\mathcal{T}}=u-v_{\mathcal{T}}$, from $\left\|h_{\mathcal{T}}\right\|_{L_{\infty}(\Omega)} \lesssim 1$ we have for $\epsilon>0$

$$
\begin{aligned}
\left\|h_{\mathcal{T} z_{T}}\right\| \|^{2} & =A\left(h_{\mathcal{T}} z_{\mathcal{T}}, h_{\mathcal{T}} z_{\mathcal{T}}\right) \\
& =\left(h_{\mathcal{T}} \nabla z_{\mathcal{T}}, \nabla\left(h_{\mathcal{T}} z_{\mathcal{T}}\right)\right)+\left(z_{\mathcal{T}} \nabla h_{\mathcal{T}}, \nabla\left(h_{\mathcal{T}} z_{\mathcal{T}}\right)\right) \\
& \leq \frac{1}{2 \epsilon}\left(\left\|h_{\mathcal{T}} \nabla z_{\mathcal{T}}\right\|_{L_{2}(\Omega)}^{2}+\left\|z_{\mathcal{T}}\right\|_{L_{2}(\Omega)}^{2}\right)+\epsilon\left\|h_{\mathcal{T}} z_{\mathcal{T}}\right\| \|^{2}
\end{aligned}
$$

or

$$
\left\|\left|h_{\mathcal{T} z \mathcal{T}}\left\|\left.\right|^{2} \lesssim\right\| h_{\mathcal{T}} \nabla z \mathcal{T}\left\|_{L_{2}(\Omega)}^{2}+\right\| z \mathcal{T} \|_{L_{2}(\Omega)}^{2}\right.\right.
$$

Integrating by parts, we next compute

$$
\begin{aligned}
\left\|h_{\mathcal{T}} \nabla z \mathcal{T}\right\|_{L_{2}(\Omega)}^{2}= & \left(\nabla z \mathcal{T}, h_{\mathcal{T}}^{2} \nabla z \mathcal{T}\right) \\
= & \sum_{T \in \mathcal{T}} \int_{T}-z \mathcal{T} \nabla \cdot\left(h_{\mathcal{T}}^{2} \nabla z \mathcal{T}\right) d x+\int_{\partial T} z \mathcal{T} h_{\mathcal{T}}^{2} \nabla z_{\mathcal{T}} \cdot \mathbf{n} \mathrm{d} \sigma \\
\leq & \sum_{T \in \mathcal{T}}\left\|z_{\mathcal{T}}\right\|_{L_{2}(T)}\left(\left\|h_{\mathcal{T}}\right\|_{L_{\infty}(T)}^{2}\left\|f+\Delta v_{\mathcal{T}}\right\|_{L_{2}(T)}\right. \\
& \left.+2\left\|\nabla h_{\mathcal{T}}\right\|_{L_{\infty}(\mathcal{T})}\left\|h_{\mathcal{T}} \nabla z \mathcal{T}\right\|_{L_{2}(T)}\right) \\
& +\left\|h_{\mathcal{T}}^{2} z \mathcal{T}\right\|_{L_{2}(\partial T \backslash \partial \Omega)}\left\|\llbracket \nabla v_{\mathcal{T}} \rrbracket\right\|_{L_{2}(\partial T \backslash \partial \Omega) .}
\end{aligned}
$$

Inserting the scaled trace inequality $\|v\|_{L_{2}(\partial T)} \lesssim h_{T}^{-1 / 2}\|v\|_{L_{2}(T)}+h_{T}^{1 / 2}\|\| v\|\|_{T}$ into (4.24) yields for $\epsilon>0$

$$
\begin{aligned}
& \left\|h_{\mathcal{T}}^{2} z_{\mathcal{T}}\right\|_{L_{2}(\partial T)}\left\|\llbracket \nabla v_{\mathcal{T}} \rrbracket\right\|_{L_{2}(\partial T)} \\
& \lesssim\left(h_{T}^{1 / 2}\left\|h_{\mathcal{T}} z_{\mathcal{T}}\right\|_{L_{2}(T)}+h_{T}^{3 / 2}\|\| h_{\mathcal{T}} z_{\mathcal{T}} \|_{T}\right)\left\|\llbracket \nabla v_{\mathcal{T}} \rrbracket\right\|_{L_{2}(\partial T)} \\
& \leq\left(\|z\|_{L_{2}(T)}+\left\|h_{\mathcal{T}} \nabla z \mathcal{T}\right\|_{L_{2}(T)}+\left\|\nabla h_{\mathcal{T}}\right\|_{L_{\infty}(T)}\left\|z_{\mathcal{T}}\right\|_{L_{2}(T)}\right) \\
& \quad \times h_{T}^{3 / 2}\left\|\llbracket \nabla v_{\mathcal{T}} \rrbracket\right\|_{L_{2}(\partial T)} \\
& \lesssim\|z\|_{L_{2}(T)}^{2}+\left(1+\frac{1}{\epsilon}\right) h_{T}^{3}\left\|\llbracket \nabla v_{\mathcal{T}} \rrbracket\right\|_{L_{2}(\partial T)}^{2}+\epsilon\left\|h_{\mathcal{T}} \nabla z_{\mathcal{T}}\right\|_{L_{2}(T)}^{2}
\end{aligned}
$$

Inserting (4.25) into (4.24) and then employing Proposition 2 and $\left.h_{\mathcal{T}}\right|_{T} \approx h_{T}$ yields for $\epsilon>0$ 


$$
\begin{aligned}
\left\|h_{\mathcal{T}} \nabla z \mathcal{T}\right\|_{L_{2}(\Omega)}^{2} & \\
\lesssim & \sum_{T \in \mathcal{T}}\left(1+\frac{1}{\epsilon}\right)\left(\|z \mathcal{T}\|_{L_{2}(T)}^{2}+h_{T}^{3}\left\|\llbracket \nabla v_{\mathcal{T}} \rrbracket\right\|_{L_{2}(\partial T \backslash \partial \Omega)}^{2}\right) \\
& +h_{T}^{4}\left\|f+\Delta v_{\mathcal{T}}\right\|_{L_{2}(T)}^{2}+\epsilon\left\|h_{\mathcal{T}} \nabla z \mathcal{T}\right\|_{L_{2}(T)}^{2} \\
\lesssim & \left(1+\frac{1}{\epsilon}\right)\left(\|z \mathcal{T}\|_{L_{2}(\Omega)}^{2}+\eta\left(v_{\mathcal{T}}, \mathcal{T}\right)^{2}\right)+\epsilon\left\|h_{\mathcal{T}} \nabla z_{\mathcal{T}}\right\|_{L_{2}(T)}^{2} \\
\lesssim & \left(1+\frac{1}{\epsilon}\right)\left(\|z \mathcal{T}\|_{L_{2}(\Omega)}^{2}+\operatorname{osc}(\mathcal{T})^{2}\right)+\epsilon\left\|h_{\mathcal{T}} \nabla z \mathcal{T}\right\|_{L_{2}(T)}^{2} .
\end{aligned}
$$

Taking $\epsilon$ small enough to kick back the last term above and inserting the result into (4.23) completes the proof.

We next prove that if $\mathcal{T}$ is sufficiently mildly graded, then $\left\|u-u_{\mathcal{T}}\right\|_{L_{2}(\Omega)}$ is bounded up to a constant by the best approximation to $u$ lying in $S_{\mathcal{T}}$, as measured in $L_{2}$, plus a data oscillation term.

Corollary 1 There exists a constant $C_{3}>0$ such that for sufficiently small $\mu$ and $\mathcal{T} \in \mathbb{T}_{\mu}$, it holds that

$$
\left\|u-u_{\mathcal{T}}\right\|_{L_{2}(\Omega)} \leq C_{3}\left[\inf _{v_{\mathcal{T}} \in S_{\mathcal{T}}}\left\|u-v_{\mathcal{T}}\right\|_{L_{2}(\Omega)}+\operatorname{osc}(\mathcal{T})\right]
$$

Proof By using that $\left\|\nabla h_{\mathcal{T}}\right\|_{L_{\infty}(\Omega)} \leq \mu$, the application of Proposition 3, Lemma 3 for some fixed $\epsilon$, and Lemma 4 shows that for any $v_{\mathcal{T}} \in S_{\mathcal{T}}$

$$
\begin{aligned}
\left\|u-u_{\mathcal{T}}\right\|_{L_{2}(\Omega)}^{2} & \lesssim\|\| h_{\mathcal{T}}\left(u-u_{\mathcal{T}}\right)\|\|^{2} \\
& \lesssim\|\| h_{\mathcal{T}}\left(u-v_{\mathcal{T}}\right)\|\|^{2}+\mu^{2}\left(\left\|u-u_{\mathcal{T}}\right\|_{L_{2}(\Omega)}^{2}+\left\|u-v_{\mathcal{T}}\right\|_{L_{2}(\Omega)}^{2}\right) \\
& \lesssim\left(1+\mu^{2}\right)\left\|u-v_{\mathcal{T}}\right\|_{L_{2}(\Omega)}^{2}+\operatorname{osc}(\mathcal{T})^{2}+\mu^{2}\left\|u-u_{\mathcal{T}}\right\|_{L_{2}(\Omega)}^{2} .
\end{aligned}
$$

By taking $\mu$ sufficiently small, the proof is completed.

Remark 3 Corollary 1 is of some interest independent of the current context because the Galerkin approximation is not stable in $L_{2}$ even on quasi-uniform meshes, that is, (4.27) does not hold if the data oscillation term is removed. A simple one-dimensional counterexample can be found in [1]. The counterexample to $L_{2}$-stability given in [1] also shows that $\left\|u-u_{\mathcal{T}}\right\|_{L_{2}(\Omega)}$ does not bound the right hand side of (4.27) up to a constant. It does however trivially follow from Corollary 1 that $\left\|u-u_{\mathcal{T}}\right\|_{L_{2}(\Omega)}+\operatorname{osc}(\mathcal{T}) \approx$ $\inf _{v_{\mathcal{T}} \in S_{\mathcal{T}}}\left\|u-v_{\mathcal{T}}\right\|_{L_{2}(\Omega)}+\operatorname{osc}(\mathcal{T})$ uniformly in all $\mathcal{T} \in \mathbb{T}_{\mu}$ with $\mu$ sufficiently small. That is, on sufficiently mildly graded meshes an analog to Céa's Lemma holds for the total $L_{2}$ error $\left\|u-u_{\mathcal{T}}\right\|_{L_{2}(\Omega)}+\operatorname{osc}(\mathcal{T})$.

\section{Adaptive FEM}

In this section we give details of our adaptive FEM. In particular, we give precise definitions of each module solve, estimate, mark, and refine of the generic 
adaptive iteration (1.2), with the goal of constructing an AFEM that produces by refinement a sequence of partitions $\left(\mathcal{T}_{i}\right)_{i \geq 0} \subset \mathbb{T}_{\mu}$ for reducing the weighted energy error $\left\|h_{i}\left(u-u_{i}\right)\right\| \|$, and thus also the $L_{2}$-error $\left\|u-u_{i}\right\|_{L_{2}(\Omega)}$. Here, and in the following, $h_{i}, u_{i}, S_{i}, \operatorname{osc}_{i}, \eta_{i}$, and, for $\mathcal{M} \subset \mathcal{T}_{i}, \eta_{i}(\mathcal{M})$ denote $h_{\mathcal{T}_{i}}, u_{\mathcal{T}_{i}}, S_{\mathcal{T}_{i}}, \operatorname{Osc}\left(\mathcal{T}_{i}\right)$, $\eta\left(u_{\mathcal{T}_{i}}, \mathcal{T}_{i}\right)$, and $\eta\left(u_{\mathcal{T}_{i}}, \mathcal{M}\right)$, respectively. The constant $\mu$ will be assumed to be sufficiently small.

1. Module solve. Given the current partition $\mathcal{T}_{i}$, solve (1.3) for $u_{i} \in S_{i}$. We assume that the finite element system is assembled and solved exactly.

2. Module estimate. In principle, the adaptive algorithm is terminated when $C \eta\left(\mathcal{T}_{i}\right) \leq t o l$ for some prescribed tolerance $t o l$ and user-defined constant $C$.

3. Module mark. We employ a Dörfler marking (cf. [6]). More precisely, we fix a parameter $0<\theta<1$, and at each step of the algorithm choose the smallest set $\mathcal{M}_{i} \subset \mathcal{T}_{i}$ so that

$$
\eta\left(\mathcal{M}_{i}\right) \geq \theta \eta_{i}
$$

4. Module refine. Our results below assume that each marked element $T \in \mathcal{M}_{i}$ is bisected $b \geq 1$ times in passing from $\mathcal{T}_{i}$ to $\mathcal{T}_{i+1}$ and that generally additional elements are refined in the process in order to ensure that $\mathcal{T}_{i+1}$ is conforming and sufficiently mildly graded in the sense that it is in $\mathbb{T}_{\mu}$. In Appendix A, we show that, assuming the value of $\mu$ is known, the standard newest vertex bisection algorithm or its generalization to more than two dimensions, as it has been implemented in the finite element toolbox ALBERTA [17], can be modified so that the sequence of adaptive meshes is indeed in $\mathbb{T}_{\mu}$. As we will see in Sect. 7 , this modification does not compromise quasi-optimality of the resulting AFEM.

\section{Convergence of the AFEM in $L_{2}$}

In this section we prove a quasi-orthogonality property, an estimator reduction inequality, and finally an error contraction property. We employ the techniques of [4], which do not rely upon local a posteriori lower bounds as do previous proofs of convergence of adaptive FEM.

Proposition 5 For any $\epsilon>0$, we have that

$$
\begin{aligned}
& \left.\left\|\left|h_{i+1}\left(u-u_{i+1}\right)\left\|\left.\right|^{2}+\right\|\right| h_{i+1}\left(u_{i+1}-u_{i}\right)\right\|\right|^{2}-(1+\epsilon)\left\|\mid h_{i}\left(u-u_{i}\right)\right\| \|^{2} \\
& \quad \lesssim \epsilon^{-1} \mu^{2}\left(\left\|u-u_{i+1}\right\|_{L_{2}(\Omega)}^{2}+\left\|u-u_{i}\right\|_{L_{2}(\Omega)}^{2}\right)
\end{aligned}
$$

Proof Writing $e_{i}=u-u_{i}$, and using that $\mathcal{T}_{i}, \mathcal{T}_{i+1} \in \mathbb{T}_{\mu}$ and $h_{i+1} \leq h_{i}$, we have

$$
\begin{aligned}
\left\|\mid h_{i+1} e_{i}\right\| & \leq\left\|h_{i+1} \nabla e_{i}\right\|_{L_{2}(\Omega)}+\left\|\nabla h_{i+1}\right\|_{L_{\infty}(\Omega)}\left\|e_{i}\right\|_{L_{2}(\Omega)} \\
& \leq\left\|h_{i} \nabla e_{i}\right\|_{L_{2}(\Omega)}+\mu\left\|e_{i}\right\|_{L_{2}(\Omega)} \leq\|\| h_{i} e_{i}\|\mid+2 \mu\| e_{i} \|_{L_{2}(\Omega)} .
\end{aligned}
$$


and so, for any $\epsilon>0$,

$$
\left.\left\|h_{i+1} e_{i}\right\|\right|^{2} \leq(1+\epsilon)\left\|\left|h_{i} e_{i}\left\|\left.\right|^{2}+\left(1+\frac{1}{\epsilon}\right) 4 \mu^{2}\right\| e_{i} \|_{L_{2}(\Omega)}^{2} .\right.\right.
$$

By substituting this result into the estimate of Lemma 3 with $\mathcal{T}=\mathcal{T}_{i+1}$, and thus $u_{\mathcal{T}}=u_{i+1}$, and $v_{\mathcal{T}}=u_{i}$, we find (6.1) with $1+\epsilon$ reading as $(1+\epsilon)^{2}$, which is an equivalent statement.

Next we establish an estimator reduction result. Our proof closely follows Corollary 3.4 of [4].

Proposition 6 With $\lambda:=1-2^{-\frac{3 b}{n}}$, for any $\delta \in(0,1]$ we have

$$
\begin{aligned}
& \eta_{i+1}^{2}-(1+\delta)\left(1-\lambda \theta^{2}\right) \eta_{i}^{2} \\
& \quad \lesssim \delta^{-1}\left[\left.\left\|h_{i+1}\left(u_{i+1}-u_{i}\right)\right\|\right|^{2}+\mu^{2}\left\|u_{i+1}-u_{i}\right\|_{L_{2}(\Omega)}^{2}\right] .
\end{aligned}
$$

Proof We first apply Lemma 1 to $u_{i}, u_{i+1} \in S_{i+1}$, square the result, and apply Young's inequality to the resulting mixed terms to obtain for any $\delta \in(0,1]$ and $T \in \mathcal{T}_{i+1}$

$$
\begin{aligned}
& \eta\left(u_{i+1}, T\right)^{2}-(1+\delta) \eta\left(u_{i}, T\right)^{2} \\
& \quad \lesssim \delta^{-1}\left[\|\| h_{i+1}\left(u_{i+1}-u_{i}\right)\left\|\left.\right|_{\tilde{\omega}_{T}} ^{2}+\right\| \nabla h_{\mathcal{T}}\left\|_{L_{\infty}(\tilde{\omega})}^{2}\right\| u_{i+1}-u_{i} \|_{L_{2}\left(\tilde{\omega}_{T}\right)}^{2}\right] .
\end{aligned}
$$

Summing over $T \in \mathcal{T}_{i+1}$ and using the fact that no element is contained in more than $n+1$ patches $\tilde{\omega}_{T}$, we have

$$
\begin{aligned}
& \eta_{i+1}^{2}-(1+\delta) \eta\left(u_{i}, \mathcal{T}_{i+1}\right)^{2} \\
& \quad \lesssim \delta^{-1}\left[\left.\left\|h_{i+1}\left(u_{i+1}-u_{i}\right)\right\|\right|^{2}+\mu^{2}\left\|u_{i+1}-u_{i}\right\|_{L_{2}(\Omega)}^{2}\right] .
\end{aligned}
$$

For $T^{\prime} \in \mathcal{T}_{i}$, let $\mathcal{T}_{T^{\prime}}=\left\{T \in \mathcal{T}_{i+1}: T \subset T^{\prime}\right\}$. Note that for a marked element $T^{\prime} \in \mathcal{M}_{i}$ and $T \in \mathcal{T}_{T^{\prime}}, h_{T} \leq 2^{-\frac{b}{n}} h_{T^{\prime}}$ and $\llbracket \nabla u_{i} \rrbracket=0$ across interfaces of $T$ lying in the interior of $T^{\prime}$, and so

$$
\eta\left(u_{i}, \mathcal{T}_{T^{\prime}}\right)^{2} \leq 2^{-\frac{3 b}{n}} \eta\left(u_{i}, T^{\prime}\right)^{2}
$$

For $T^{\prime} \in \mathcal{T}_{i} \backslash \mathcal{M}_{i}$, we combine (6.3) with the trivially proved monotonicity property $\eta\left(u_{i}, \mathcal{T}_{T^{\prime}}\right) \leq \eta\left(u_{i}, T^{\prime}\right)$ and sum over $T \in \mathcal{T}_{i+1}$ to obtain

$$
\begin{aligned}
\eta\left(u_{i}, \mathcal{T}_{i+1}\right)^{2} \leq & \eta\left(u_{i}, \mathcal{T}_{i} \backslash \mathcal{M}_{i}\right)^{2}+2^{-\frac{3 b}{n}} \eta\left(u_{i}, \mathcal{M}_{i}\right)^{2} \\
& =\eta\left(u_{i}, \mathcal{T}_{i}\right)^{2}-\lambda \eta\left(u_{i}, \mathcal{M}_{i}\right)^{2} \leq\left(1-\lambda \theta^{2}\right) \eta_{i}^{2}
\end{aligned}
$$

by (5.1). Combining (6.2) and (6.4) completes the proof. 
We finally establish a contraction property for $\left\|\left|h_{i}\left(u-u_{i}\right) \|\right|^{2}+\gamma \eta\left(\mathcal{T}_{i}\right)^{2}\right.$ for some properly chosen constant $\gamma>0$.

Theorem 2 There exist constants $\gamma>0$ and $\alpha \in(0,1)$ depending on a generic constant of the type defined in Sect. 2.4, the parameter $\theta$ in (5.1), and the number of times $b$ that each element in $\mathcal{M}_{i}$ is bisected such that for sufficiently small $\mu$,

$$
\left\|\left|h_{i+1}\left(u-u_{i+1}\right) \|\right|^{2}+\gamma \eta_{i+1}^{2} \leq \alpha^{2}\left(\left\|\mid h_{i}\left(u-u_{i}\right)\right\| \|^{2}+\gamma \eta_{i}^{2}\right) .\right.
$$

Proof We will use the abbreviations $e_{j}=\|\| h_{j}\left(u-u_{j}\right)\|\|(j \in\{i, i+1\})$, and $E_{i}=\left\|h_{i+1}\left(u_{i}-u_{i+1}\right)\right\| \|$.

From Propositions 1 and 4-6, we know that there exists constants $C_{4}, C_{5}, C_{6}>0$ such that for all $\epsilon, \delta>0$,

$$
\begin{aligned}
e_{i} \leq & C_{4} \eta_{i} \\
e_{i+1}^{2} \leq & (1+\epsilon) e_{i}^{2}-E_{i}^{2}+C_{5} \epsilon^{-1} \mu^{2}\left(\eta_{i}^{2}+\eta_{i+1}^{2}\right) \\
\eta_{i+1}^{2} \leq & (1+\delta)\left(1-\lambda \theta^{2}\right) \eta_{i}^{2}+C_{6} \delta^{-1}\left[E_{i}+\mu^{2}\left(\eta_{i}^{2}+\eta_{i+1}^{2}\right)\right] \\
\leq & (1+\delta)\left[\left(1-\frac{1}{2} \lambda \theta^{2}\right) \eta_{i}^{2}-\frac{1}{2 C_{4}^{2}} \lambda \theta^{2} e_{i}^{2}\right] \\
& +C_{6} \delta^{-1}\left[E_{i}+\mu^{2}\left(\eta_{i}^{2}+\eta_{i+1}^{2}\right)\right]
\end{aligned}
$$

where to arrive at (6.8) we used already (6.6).

Multiplying (6.8) with $\tilde{\gamma}=\tilde{\gamma}(\delta)=\delta C_{6}^{-1}$ and adding the result to (6.7) yields

$$
\begin{aligned}
& e_{i+1}^{2}+\tilde{\gamma} \eta_{i+1}^{2} \leq(1+\delta)\left(1-\frac{1}{2} \lambda \theta^{2}\right) \tilde{\gamma} \eta_{i}^{2} \\
& +\left[(1+\epsilon)-(1+\delta) \frac{\tilde{\gamma}}{2 C_{4}^{2}} \lambda \theta^{2}\right] e_{i}^{2}+q \mu^{2}\left(\eta_{i}^{2}+\eta_{i+1}^{2}\right),
\end{aligned}
$$

where $q=q(\epsilon)=C_{5} \epsilon^{-1}+1$. Now by fixing a sufficiently small $\delta$ and, subsequently, a sufficiently small $\epsilon$ such that

$$
\tilde{\alpha}^{2}:=\max \left\{(1+\delta)\left(1-\frac{1}{2} \lambda \theta^{2}\right),(1+\epsilon)-(1+\delta) \frac{\tilde{\gamma}}{2 C_{4}^{2}} \lambda \theta^{2}\right\}<1,
$$

(6.10) implies that

$$
e_{i+1}^{2}+\left(\tilde{\gamma}-q \mu^{2}\right) \eta_{i+1}^{2} \leq \tilde{\alpha}^{2} e_{i}^{2}+\left(\tilde{\alpha}^{2} \tilde{\gamma}+q \mu^{2}\right) \eta_{i}^{2}
$$


Now by choosing $\mu$ sufficiently small such that $0<\frac{\tilde{\alpha}^{2} \tilde{\gamma}+q \mu^{2}}{\tilde{\gamma}-q \mu^{2}} \leq \alpha^{2}:=\frac{1+\tilde{\alpha}^{2}}{2}$, the proof is completed for that $\alpha$ and $\gamma:=\tilde{\gamma}-q \mu^{2}$.

We finally show that $\left\|u-u_{i}\right\|_{L_{2}(\Omega)} \rightarrow 0$ with linear rate as $i \rightarrow \infty$.

Corollary 2 Assume that $\mu$ is sufficiently small as in Theorem 2. Then with $\alpha$ from that theorem, it holds that for $i \geq j$

$$
\left\|u-u_{i}\right\|_{L_{2}(\Omega)}+\operatorname{osc}_{i} \lesssim \alpha^{i-j}\left[\left\|u-u_{j}\right\|_{L_{2}(\Omega)}+\operatorname{osc}_{j}\right]
$$

Proof The proof follows from Theorem 2 and

$$
\left(\left\|u-u_{i}\right\|_{L_{2}(\Omega)}+\operatorname{osc}_{i}\right)^{2} \approx\left\|\left|h_{i}(u-u) \|\right|^{2}+\gamma \eta_{i}^{2}\right.
$$

the latter being a consequence of Propositions 1, 2 and 4, and Remark 1.

\section{Quasi-optimality of AFEM in $L_{2}$}

For $s>0$, we define the approximation class

$$
\begin{aligned}
\mathcal{A}^{s} & =\left\{v \in H_{0}^{1}(\Omega):-\Delta v \in L_{2}(\Omega),|v|_{\mathcal{A}^{s}}\right. \\
& \left.:=\sup _{N \in \mathbb{N}} N^{s} \inf _{\left\{\mathcal{T} \in \mathbb{T}: \# \mathcal{T}-\# \mathcal{T}_{0} \leq N\right\}}\left[\inf _{v_{\mathcal{T}} \in S_{\mathcal{T}}}\left\|u-v_{\mathcal{T}}\right\|_{L_{2}(\Omega)}+\operatorname{osc}(\mathcal{T})\right]<\infty\right\} .
\end{aligned}
$$

Thus $\mathcal{A}^{s}$ contains all $v \in H_{0}^{1}(\Omega)$ with $-\Delta v \in L_{2}(\Omega)$, that, for some sequence $\left(\mathcal{T}_{i}\right)_{i}$, can be approximated at rate $s$ in the $L_{2}$-norm by a sequence of functions from $\left(S_{\mathcal{T}_{i}}\right)_{i}$, and for which $-\Delta v$ can be approximated by a sequence of functions from $\left(\prod_{T \in \mathcal{T}_{i}} \mathbb{P}_{k-1}(T)\right)_{i}$ in the $\mathcal{T}_{i}$-dependent weighted $L_{2}$-norm $\sqrt{\sum_{T \in \mathcal{T}_{i}} h_{T}^{4}\|g\|_{L_{2}(T)}^{2}}$.

Note that in our case of $\Omega$ being convex, $v \in H_{0}^{1}(\Omega)$ with $-\Delta v \in L_{2}(\Omega)$ is equivalent to $v \in H_{0}^{1}(\Omega) \cap H^{2}(\Omega)$. Standard estimates show that for $s \in\left[\frac{2}{n}, \frac{k+1}{n}\right]$, we have $H_{0}^{1} \cap H^{s n}(\Omega) \subset \mathcal{A}^{s}$, where for $v \in H_{0}^{1} \cap H^{s n}(\Omega)$ the rate $s$ is already realized with uniform refinements. The class $\mathcal{A}^{s}$, however, is much larger than $H_{0}^{1} \cap H^{s n}(\Omega)$, which is the reason an AFEM is employed in the first place. What is in essence needed for $v \in H_{0}^{1} \cap H^{2}(\Omega)$ to be in $\mathcal{A}^{s}$ is that its $s n$th order partial derivatives are bounded in $L_{p}(\Omega)$ for some $p>\left(\frac{1}{2}+s\right)^{-1}$.

In this section, we are going to show that if, for whatever $s>0$, the solution $u$ of (1.1) is in $\mathcal{A}^{s}$, then for the sequence $\left(\mathcal{T}_{i}\right)_{i} \subset \mathbb{T}_{\mu}$ and corresponding Galerkin solutions $\left(u_{i}\right)_{i}$ produced by our AFEM, it holds that $\left\|u-u_{i}\right\|_{L_{2}(\Omega)}+\operatorname{osc}_{i} \lesssim\left(\# \mathcal{T}_{i}-\# \mathcal{T}_{0}\right)^{-s}|u|_{\mathcal{A}^{s}}$. Thus our AFEM realizes the best possible convergence rate.

For earlier results on quasi-optimality of AFEM with respect to the energy-norm and more details on approximation classes and references, we refer to $[4,16,19]$.

The following lemma will be used to bound the number of marked elements in the Dörfler marking. 
Lemma 5 With $C_{1}$ and $C_{2}$ being the constants from Proposition 2 and Lemma 2, let $\theta<\frac{1}{C_{1}\left(C_{2}+1\right)}$. Let $\mu$ be sufficiently small such that Lemma 2 is valid. Then for $\left(\mathbb{T}_{\mu} \ni\right) \mathcal{T}_{i} \subset \mathcal{T} \in \mathbb{T}$ with

$$
\left\|u-u_{\mathcal{T}}\right\|_{L_{2}(\Omega)}+\operatorname{osc}(\mathcal{T}) \leq\left[1-\theta C_{1}\left(C_{2}+1\right)\right]\left(\left\|u-u_{i}\right\|_{L_{2}(\Omega)}+\operatorname{osc}_{i}\right),
$$

it holds that

$$
\eta\left(\mathcal{R}_{\mathcal{T}_{i} \rightarrow \mathcal{T}}\right) \geq \theta \eta_{i}
$$

Proof By adding the inequalities

$$
\begin{aligned}
\left\|u-u_{i}\right\|_{L_{2}(\Omega)} & \leq\left\|u_{i}-u_{\mathcal{T}}\right\|_{L_{2}(\Omega)}+\left\|u-u_{\mathcal{T}}\right\|_{L_{2}(\Omega)} \\
\operatorname{osc}_{i} & \leq \operatorname{osc}\left(\mathcal{R}_{\mathcal{T}_{i} \rightarrow \mathcal{T}}\right)+\operatorname{osc}(\mathcal{T})
\end{aligned}
$$

and employing (7.1), we infer that

$$
\begin{aligned}
\theta\left(C_{2}+1\right) \eta_{i} & \leq \theta C_{1}\left(C_{2}+1\right)\left(\left\|u-u_{i}\right\|_{L_{2}(\Omega)}+\operatorname{osc}_{i}\right) \\
& \leq\left\|u-u_{i}\right\|_{L_{2}(\Omega)}+\operatorname{osc}_{i}-\left\|u-u_{\mathcal{T}}\right\|_{L_{2}(\Omega)}-\operatorname{osc}(\mathcal{T}) \\
& \leq\left\|u_{i}-u_{\mathcal{T}}\right\|_{L_{2}(\Omega)}+\operatorname{osc}\left(\mathcal{R}_{\mathcal{T}_{i} \rightarrow \mathcal{T}}\right) \\
& \leq\left(C_{2}+1\right) \eta\left(\mathcal{R}_{\left.\mathcal{T}_{i} \rightarrow \mathcal{T}\right)},\right.
\end{aligned}
$$

where the first and last inequality follow from applications of Proposition 2 and Lemma 2, respectively.

Corollary 3 For some $s>0$, let $u \in \mathcal{A}^{s}$. Assume also that $\mu$ is sufficiently small that Corollary 1 is valid. Then under the assumptions of Lemma 5, the collection of marked elements $\mathcal{M}_{i} \subset \mathcal{T}_{i}$ defined by (5.1) satisfies

$$
\# \mathcal{M}_{i} \lesssim|u|_{\mathcal{A}^{s}}^{1 / s}\left(\left\|u-u_{i}\right\|_{L_{2}(\Omega)}+\operatorname{osc}_{i}\right)^{-1 / s}
$$

Proof With $C_{3}$ the constant from Corollary 1, by definition of $\mathcal{A}^{s}$ there exists a partition $\mathcal{T}^{\prime} \in \mathbb{T}$ with

$$
\# \mathcal{T}^{\prime}-\# \mathcal{T}_{0} \lesssim|u|_{\mathcal{A}^{s}}^{1 / s}\left(\frac{1-\theta C_{1}\left(C_{2}+1\right)}{1+C_{3}}\left(\left\|u-u_{i}\right\|_{L_{2}(\Omega)}+\operatorname{osc}_{i}\right)\right)^{-1 / s}
$$

and a $v_{\mathcal{T}^{\prime}} \in S_{\mathcal{T}}$, with

$$
\left\|u-v_{\mathcal{T}^{\prime}}\right\|_{L_{2}(\Omega)}+\operatorname{osc}\left(\mathcal{T}^{\prime}\right) \leq \frac{1-\theta C_{1}\left(C_{2}+1\right)}{1+C_{3}}\left(\left\|u-u_{i}\right\|_{L_{2}(\Omega)}+\operatorname{osc}_{i}\right) .
$$

As is shown in Appendix A, $\mathcal{T}^{\prime}$ can be refined to a partition $\mathcal{T}^{\prime \prime} \in \mathbb{T}_{\mu}$ with $\# \mathcal{T}^{\prime \prime}-\# \mathcal{T}_{0} \lesssim$ $\# \mathcal{T}^{\prime}-\# \mathcal{T}_{0}$, dependent on $\mu$. The smallest common refinement $\mathcal{T}$ of $\mathcal{T}_{i}$ and $\mathcal{T}^{\prime \prime}$ is in 
$\mathbb{T}_{\mu}$ with $\# \mathcal{T}-\# \mathcal{T}_{i} \leq \# \mathcal{T}^{\prime \prime}-\# \mathcal{T}_{0}$ (cf. [19] last lines of the proof of Lemma 5.2). Since $S_{\mathcal{T}} \subset S_{\mathcal{T}^{\prime}}$, Corollary 1 shows that

$$
\begin{aligned}
\left\|u-u_{\mathcal{T}}\right\|_{L_{2}(\Omega)}+\operatorname{osc}(\mathcal{T}) & \leq\left(C_{3}+1\right)\left(\inf _{v_{\mathcal{T}^{\prime}} \in S_{\mathcal{T}^{\prime}}}\left\|u-v_{\mathcal{T}^{\prime}}\right\|_{L_{2}(\Omega)}+\operatorname{osc}\left(\mathcal{T}^{\prime}\right)\right) \\
& \leq\left(1-\theta C_{1}\left(C_{2}+1\right)\right)\left(\left\|u-u_{i}\right\|_{L_{2}(\Omega)}+\operatorname{osc}_{i}\right),
\end{aligned}
$$

and so $\eta\left(\mathcal{R}_{\mathcal{T}_{i} \rightarrow \mathcal{T}}\right) \geq \theta \eta_{i}$ by Lemma 5. Since $\mathcal{M}_{i}$ is the smallest subset of $\mathcal{T}_{i}$ with $\eta\left(\mathcal{M}_{i}\right) \geq \theta \eta_{i}$, we conclude that

$$
\# \mathcal{M}_{i} \leq \# \mathcal{R}_{\mathcal{T}_{i} \rightarrow \mathcal{T}} \leq \# \mathcal{T}-\# \mathcal{T}_{i} \leq \# \mathcal{T}^{\prime \prime}-\# \mathcal{T}_{0} \lesssim \# \mathcal{T}^{\prime}-\# \mathcal{T}_{0}
$$

so that the proof follows from (7.2).

Finally, in the next theorem the quasi-optimality result is stated.

Theorem 3 For some $s>0$, let $u \in \mathcal{A}^{s}$. Then, under the assumptions from Corollary 3, it holds that

$$
\# \mathcal{T}_{i}-\# \mathcal{T}_{0} \lesssim\left(\left\|u-u_{i-1}\right\|_{L_{2}(\Omega)}+\operatorname{osc}_{i-1}\right)^{-1 / s}|u|_{\mathcal{A}^{s}}^{1 / s}
$$

Proof In Theorem 4 it is shown that $\# \mathcal{T}_{i}-\# \mathcal{T}_{0} \lesssim \sum_{j=0}^{i-1} \mathcal{M}_{j}$. The proof follows by combining this result with Corollaries 2 and 3.

\section{Numerical example}

In this section we discuss the relationship between AFEM for controlling $L_{2}$ and energy norms and illustrate our results via a computational example. This example highlights two interesting aspects of AFEM convergence theory. First, one generally cannot expect simultaneous optimal control of the error in two different norms such as the $L_{2}$ and $H^{1}$ norms. Secondly, in contrast to the usual intuition about the relationship between $L_{2}$ and energy convergence rates, an $L_{2}$ AFEM can produce a convergence rate which is more than $\frac{1}{n}$ greater than the best possible convergence rate for an energy AFEM for the same problem.

As an example problem, we let $\Omega$ be a convex polyhedron in $\mathbb{R}^{3}$ having maximum edge opening angle $\frac{7 \pi}{8}$ on a given edge $e_{\max }$. In our computational example we took $\Omega$ to be the union of two tetrahedron, though the precise nature of $\Omega$ is not essential so long as the maximum edge opening angle is controlled. We then solve Poisson's problem (1.1) on $\Omega$ with right hand side data $f=1$. By standard theory, we expect the solution $u$ to have an edge singularity of the form $r^{\frac{8}{7}}$, where $r$ is the distance to the edge $e_{\text {max }}$. In our example we also employed polynomial degree $k=4$. Finally, the Dörfler marking parameter $\theta$ was taken to be 0.4 . Because AFEM optimality requires that $\theta$ be small enough, we also tried lower values of $\theta$ but observed no appreciable difference in convergence rates when doing so. Calculations were carried out using the finite element toolbox ALBERTA (cf. [17]). 
We now describe the implications of these choices. Following the discussion in Sect. 7, we expect our $L_{2}$ AFEM to converge with the rate $s=\frac{k+1}{n}$ so long as the $k+1$-st (and thus $s n$-th) order partial derivatives are $p$-integrable for some $p>\left(\frac{1}{2}+s\right)^{-1}$. Note that because $f=1$, no data oscillation is present in this problem, so the $L_{2}$ error is in fact equivalent to the error estimator and the approximation class $\mathcal{A}^{s}$ can be defined only in terms of the error. A precise characterization of $\mathcal{A}^{s}$ in terms of Besov spaces is also possible in this case, though heuristics phrased in terms of $L_{p}$ spaces are sufficient for our purposes here. In our example we have $k+1=5$ and $n=3$, so for a convergence rate of $\frac{5}{3}$ we roughly require that $D^{5} u \in L_{p}(\Omega)$ with $p>\frac{6}{13}$. Our example problem has been constructed so that the singularity $r^{\frac{8}{7}}$ is the strongest occurring in the solution, and it is easy to calculate that $D^{5} r^{\frac{8}{7}} \approx r^{\frac{8}{7}-5}$ indeed is $p$-integrable for some values of $p>\frac{6}{13}$.

We now compare the expected $L_{2}$ convergence rate with the expected $H^{1}$ convergence rate for our example problem. A standard $H^{1}$ AFEM will converge with rate $s \in\left[\frac{1}{n}, \frac{k}{n}\right]$ when roughly speaking $u \in H_{0}^{1}(\Omega)$ with its $s n+1$-th partial derivatives lying in $L_{p}$ for $p>\left(\frac{1}{2}+s\right)^{-1}$. Thus for polynomial degree $k=4$, the generally best possible convergence rate of $\frac{4}{3}$ can be obtained if $D^{5} u \in L_{p}(\Omega)$ for some $p>\frac{6}{11}$. Note that this condition is more stringent than that required above for obtaining the generally best possible convergence rate of $\frac{5}{3}$ in $L_{2}$, and in fact for our example problem it is easy to calculate that $D^{5} r^{\frac{8}{7}}$ does not lie in $L_{p}$ for any $p>\frac{6}{11}$. Solving the conditions $D^{\alpha+1} r^{\frac{8}{7}} \in L_{p}(\Omega)$ with $\alpha=s n$ and $p>\left(\frac{1}{2}+s\right)^{-1}$ yields $s<\frac{8}{7}$, so we predict that a standard $H^{1}$ AFEM will converge with rate $s<\frac{8}{7} \approx 1.14$.

In our tests we ran separate computations using $L_{2}$ error indicators in the mark step and using $H^{1}$ error indicators in the mark step. In both cases, we recorded both the $L_{2}$ and $H^{1}$ error estimators, which are uniformly equivalent to the actual respective errors because no data oscillation is present. The results of these tests can be seen in Fig. 1. Approximate convergence rates were calculated by a least squares fit of logarithmic data over the range from $6 \times 10^{6}$ to $1.5 \times 10^{8}$ degrees of freedom, and corresponding trend lines are displayed on the plot along with computed data.

We note several interesting features of this plot. First, the computed convergence rate of $s=1.65$ in the $L_{2}$ norm when using the $L_{2}$ AFEM is very close to the predicted optimal rate of $\frac{5}{3}$, while the computed convergence rate in the energy norm of 1.12 when using an energy AFEM is similarly close to the maximum predicted rate of $\frac{8}{7}$ for this particular problem. Thus as predicted by AFEM convergence theory, our $L_{2}$ AFEM produces a convergence rate which is more than $\frac{1}{n}$ greater than the best possible convergence rate for an energy AFEM for the same problem.

We next discuss convergence rates in the $L_{2}$ norm when energy refinement is used, and vice versa. When energy refinement is employed, we obtain a convergence rate $s=1.47$ for the $L_{2}$ error, which is roughly $\frac{1}{3}=\frac{1}{n}$ more than the observed energy convergence rate of 1.12. Thus refining using energy indicators will not in all cases lead to optimal convergence in the $L_{2}$ norm. This difference is due to the fact that in 


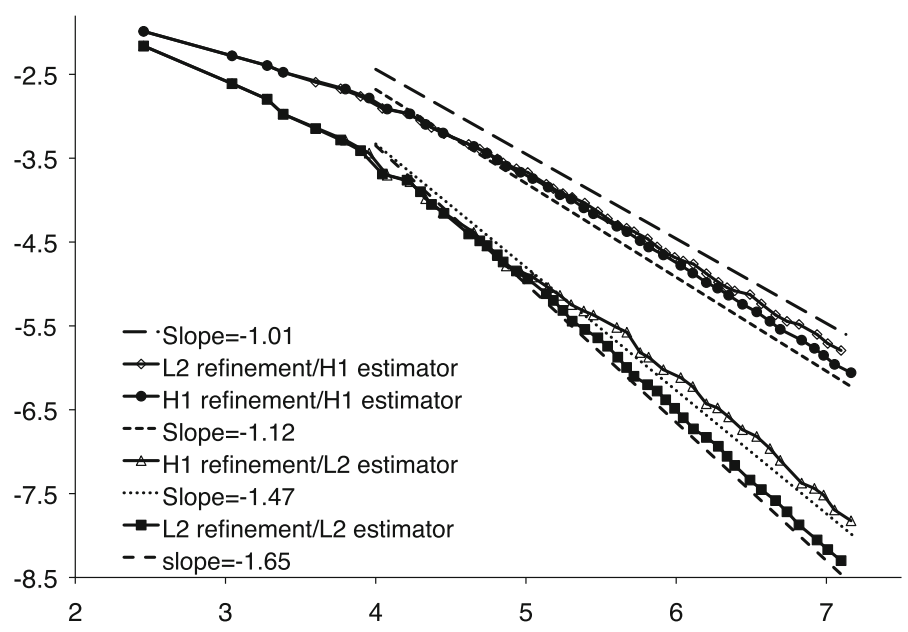

Fig. 1 Error reduction in the $L_{2}$ and energy norms when refining using $H^{1}$ error indicator ("H1 refinement") and $L_{2}$ error indicators "L2 refinement"); plot shows $\log ($ estimator) versus $\log ($ DOF $)$

general more severe local refinement is necessary to obtain the best possible energy convergence rate than is necessary to obtain the best possible $L_{2}$ convergence rate. One would correspondingly expect that $L_{2}$ refinement will sometimes lead to suboptimal energy error decrease, and in our example we in fact observe a slight decrease in energy convergence rate (from 1.12 to 1.01 ) when using $L_{2}$ instead of energy refinement. Thus we conclude that simultaneous optimal error control in multiple norms is not generally possible.

We close this section with two concluding remarks about our experiments.

Remark 4 In our experiments we employed the standard refinement procedure implemented in ALBERTA, and in particular did not take extra steps to ensure that the meshes remained sufficiently mildly graded as is required in our theoretical results. We are not aware of any experimental evidence that sufficiently mild grading is necessary in practice.

Remark 5 Two-dimensional examples can be constructed for which convergence order $\frac{k+1}{2}$ is expected in the $L_{2}$ norm, but where the optimal $H^{1}$ AFEM produces a convergence rate of less than $\frac{k}{2}$. However, in contrast to the three-dimensional case such examples require a singular right-hand-side $f$ since corner singularities have infinite smoothness in the relevant scales of Besov spaces. As an example, take $\Omega$ to be the unit square, and let $f(x, y)=x^{-0.4}$. Theoretical considerations similar to those above lead us to expect that for polynomial degree $k=3$, the $L_{2}$ AFEM will lead to convergence rate 2 in the $L_{2}$ norm, while convergence in the $H^{1}$ norm when using the energy AFEM is expected to be less than $\frac{3}{2}$. Coding such examples in a meaningful way is not entirely straightforward, however, because accurate quadrature for singular functions must be constructed and data oscillation is also present and plays an important role in the convergence theory. 
Open Access This article is distributed under the terms of the Creative Commons Attribution Noncommercial License which permits any noncommercial use, distribution, and reproduction in any medium, provided the original author(s) and source are credited.

\section{Appendix A: Partitions and mesh functions}

We specify the type of partitions that we consider, and derive some of their properties. When doing so we recall some results from [20], which generalize upon known results for newest vertex bisection in two dimensions. In addition, we introduce the concept of sufficiently mildly graded partitions and define suitable mesh-functions.

\section{A.1 Bisection and uniform shape regularity}

For $0 \leq p \leq n-1$, a (closed) simplex spanned by $p+1$ vertices of an $n$-simplex $T$ is called a hyperface of $T$. For $p=n-1$, it will be called a facet. A partition $\mathcal{T}$ of a domain $\Omega \subset \mathbb{R}^{n}$, i.e., a subdivision of $\Omega$ into (essentially) disjoint closed $n$-simplices, is called conforming when the intersection of any two different $T, T^{\prime} \in \mathcal{T}$ is either empty, or a hyperface of both simplices. Different simplices $T, T^{\prime}$ that share a facet will be called neighbors.

Simplices are refined by means of bisection. In order to guarantee uniform shape regularity of all descendants, a cyclic choice of the refinement edges has to be made. To that end, given $\left\{x_{0}, \ldots x_{n}\right\} \subset \mathbb{R}^{n}$, not on a joint $(n-1)$-dimensional hyperplane, we distinguish between $n(n+1)$ ! tagged simplices given by all possible ordered sequences $\left(x_{0}, x_{1}, \ldots, x_{n}\right)_{\gamma}$ and types $\gamma \in\{0, \ldots, n-1\}$. Given a tagged simplex $T=\left(x_{0}, x_{1}, \ldots, x_{n}\right)_{\gamma}$, its children are the tagged simplices

$$
\left(x_{0}, \frac{x_{0}+x_{n}}{2}, x_{1}, \ldots, x_{\gamma}, x_{\gamma+1}, \ldots, x_{n-1}\right)_{(\gamma+1) \bmod n}
$$

and

$$
\left(x_{n}, \frac{x_{0}+x_{n}}{2}, x_{1}, \ldots, x_{\gamma}, x_{n-1}, \ldots, x_{\gamma+1}\right)_{(\gamma+1) \bmod n},
$$

where the sequences $\left(x_{\gamma+1}, \ldots, x_{n-1}\right)$ and $\left(x_{1}, \ldots, x_{\gamma}\right)$ should be read as being void for $\gamma=n-1$ and $\gamma=0$, respectively. So these children are defined by bisecting the edge $\overline{x_{0} x_{n}}$ of $T$, i.e., by connecting its midpoint with the other vertices $x_{1}, \ldots, x_{n-1}$, and by an appropriate ordering of their vertices, and by having type $(\gamma+1) \bmod n$ (see Fig. 2 for an illustration). This bisection process was introduced in [21], and in different notations, in [10]. The edge $\overline{x_{0} x_{n}}$ is called the refinement edge of $T$. In the $n=2$ case, the vertex opposite to this edge is known as the newest vertex.

Corresponding to a tagged simplex $T=\left(x_{0}, \ldots, x_{n}\right)_{\gamma}$, we set

$$
T_{\mathrm{R}}=\left(x_{n}, x_{1}, \ldots, x_{\gamma}, x_{n-1}, \ldots, x_{\gamma+1}, x_{0}\right)_{\gamma},
$$



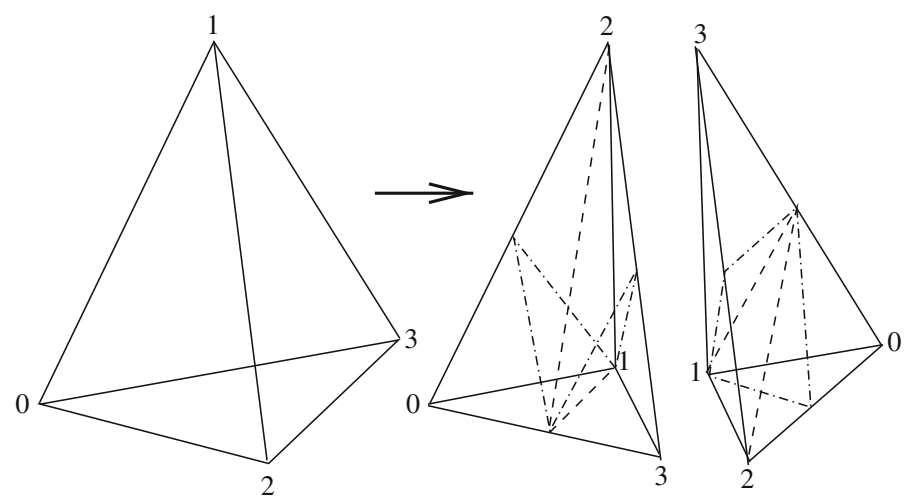

Fig. 2 Bisection of a tagged tetrahedron of type 0 with the next two level cuts indicated

which is the tagged simplex that has the same set of children as $T$, and in this sense is equal to $T$. So actually we distinguish between $\frac{1}{2} n(n+1)$ ! tagged simplices.

Given a fixed, conforming initial partition $\mathcal{T}_{0}$ of $\bar{\Omega}$ into tagged simplices of some fixed type $\gamma$, we exclusively consider partitions that can be created from $\mathcal{T}_{0}$ by recurrent bisections of tagged simplices, for short, descendants of $\mathcal{T}_{0}$. Simplices from descendants of $\mathcal{T}_{0}$ are uniformly shape regular, dependent only on $\mathcal{T}_{0}$ and $n$. In view of the refinement by bisection, this means that there exist constants $d, D>0$, dependent only on $\mathcal{T}_{0}$, such that

$$
d 2^{-\ell(T)} \leq \operatorname{vol}(T)\left(=h_{T}^{n}\right), \quad \operatorname{diam}(T) \leq D 2^{-\ell(T) / n},
$$

Here $\ell(T)$ denotes the level of a $T$, being the number of bisections needed to create $T$ from a simplex from $\mathcal{T}_{0}$.

\section{A.2 Conforming partitions}

For the application of an a posteriori error estimator, we need partitions that are conforming. The set of conforming descendants of $\mathcal{T}_{0}$ is denoted as $\mathbb{T}$. A partition generated from a conforming one by bisecting some marked simplices is generally non-conforming, so that additional bisections have to be made to restore conformity.

To bound the cardinality of the output partition of a call of AFEM, we would like that the total number of additional bisections needed to restore conformity after bisecting some marked simplices can be bounded by some absolute multiple of the total number of marked simplices inside such a call. To guarantee this property, we assume that the simplices from $\mathcal{T}_{0}$ are tagged in such a way that any two neighbors $T=\left(x_{0}, \ldots, x_{n}\right)_{\gamma}, T^{\prime}=\left(x_{0}^{\prime}, \ldots, x_{n}^{\prime}\right)_{\gamma}$ from $\mathcal{T}_{0}$ match in the sense that if $\overline{x_{0} x_{n}}$ or $\overline{x_{0}^{\prime} x_{n}^{\prime}}$ is on $T \cap T^{\prime}$, then either $T$ and $T^{\prime}$ are reflected neighbors-meaning that the ordered sequence of vertices of either $T$ or $T_{\mathrm{R}}$ coincides with that of $T^{\prime}$ on all but one position-or the pair of neighboring children of $T$ and $T^{\prime}$ are reflected neighbors (see Fig. 3 for an illustration). 

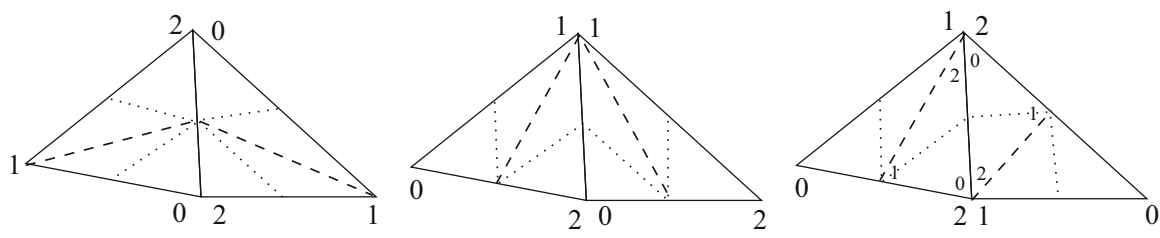

Fig. 3 Matching neighbors for $n=2$, and their level 1 and 2 descendants. The neighbors in the rightmost picture are not reflected neighbors, but the pair of their neighboring children are

It is known (see [2] and the references therein) that for any conforming partition into triangles, i.e., for $n=2$, there exists a local numbering of the vertices so that the matching condition is satisfied. For $n>2$, any conforming partition of $n$-simplices can be refined, by inflating the number of simplices by not more than an absolute constant factor, into a conforming partition $\mathcal{T}_{0}$ that allows a local numbering of the vertices so that the matching condition is satisfied.

The matching condition on $\mathcal{T}_{0}$ is a necessary and sufficient condition so that any uniform refinement of $\mathcal{T}_{0}$-i.e., a refinement of $\mathcal{T}_{0}$ in which all simplices have the same level-is conforming. For this reason, we always assume that $\mathcal{T}_{0}$ satisfies the matching condition.

Tagged neighbors are called compatibly divisible when they have the same refinement edge. For a descendant $\mathcal{T}$ of $\mathcal{T}_{0}$, and $T \in \mathcal{T}$, we set

$$
\begin{aligned}
& N(\mathcal{T}, T):= \\
& \quad \text { neighbors } T^{\prime} \text { of } T \text { in } \mathcal{T} \text { that contain the refinement edge of } T \text { \}. }
\end{aligned}
$$

As a consequence of the matching condition, we have the following result.

Corollary 4 For any conforming descendant $\mathcal{T}$ of $\mathcal{T}_{0}$, and $T \in \mathcal{T}$, it holds that

1. $\left|\ell(T)-\ell\left(T^{\prime}\right)\right| \leq 1$ for any neighbor $T^{\prime} \in \mathcal{T}$ of $T$,

2. and for $T^{\prime} \in N(\mathcal{T}, T)$, either

- $\ell\left(T^{\prime}\right)=\ell(T)$ and $T, T^{\prime}$ are compatibly divisible, or

- $\ell\left(T^{\prime}\right)=\ell(T)-1$ and $T$ is compatibly divisible with one of both children of $T^{\prime}$.

In view of the second part of this corollary, an algorithm bisect $[\mathcal{T}, T]$ for finding the smallest $\mathcal{T} \subset \mathcal{T}^{\prime} \in \mathbb{T}$ in which some selected simplex $T \in \mathcal{T}$ is bisected may consist of the following two steps: firstly, by recursive calls of bisect[ $\left.\mathcal{T}, T^{\prime \prime}\right]$ for some $T^{\prime \prime}$ with $\ell\left(T^{\prime \prime}\right)=\ell(T)-1$, construct a possibly refined partition $\mathcal{T} \subseteq \mathcal{T}^{\prime} \in \mathbb{T}$ that contains a subset $T \in K \subset \mathcal{T}^{\prime}$ so that all $T^{\prime} \in K$ share their refinement edge with $T$. Secondly, bisect all these $T^{\prime} \in K$ simultaneously, with which thus no "hanging nodes" are created.

\section{A.3 Graded partitions and the routine "bisect"}

For purposes of proving convergence and optimality of $L_{2}$ AFEM, we need partitions $\mathcal{T} \in \mathbb{T}$ whose (possible) grading is sufficiently mild. Given a constant $G>0$, we call 
a descendant $\mathcal{T}$ of $\mathcal{T}_{0} G$-graded when for all $T, T^{\prime} \in \mathcal{T}$,

$$
\operatorname{dist}\left(T, T^{\prime}\right) \leq G 2^{-\ell(T) / n} \Longrightarrow \ell\left(T^{\prime}\right) \geq \ell(T)-1 .
$$

Thus $G$-gradedness locally restricts the growth of the size of mesh elements as one moves away from a given element. If we want to bisect a simplex in such a partition, then generally additional bisections have to be made not only to guarantee conformity of the resulting partition, but also its $G$-gradedness. The following routine bisect $[\mathcal{T}, T]$ ensures both properties. The first part of this routine, whose steps were already described at the end of the previous section, can be found in [20], whereas its last, additional loop before the actual bisection of $T$ in the last line ensures the $G$-gradedness of the output partition.

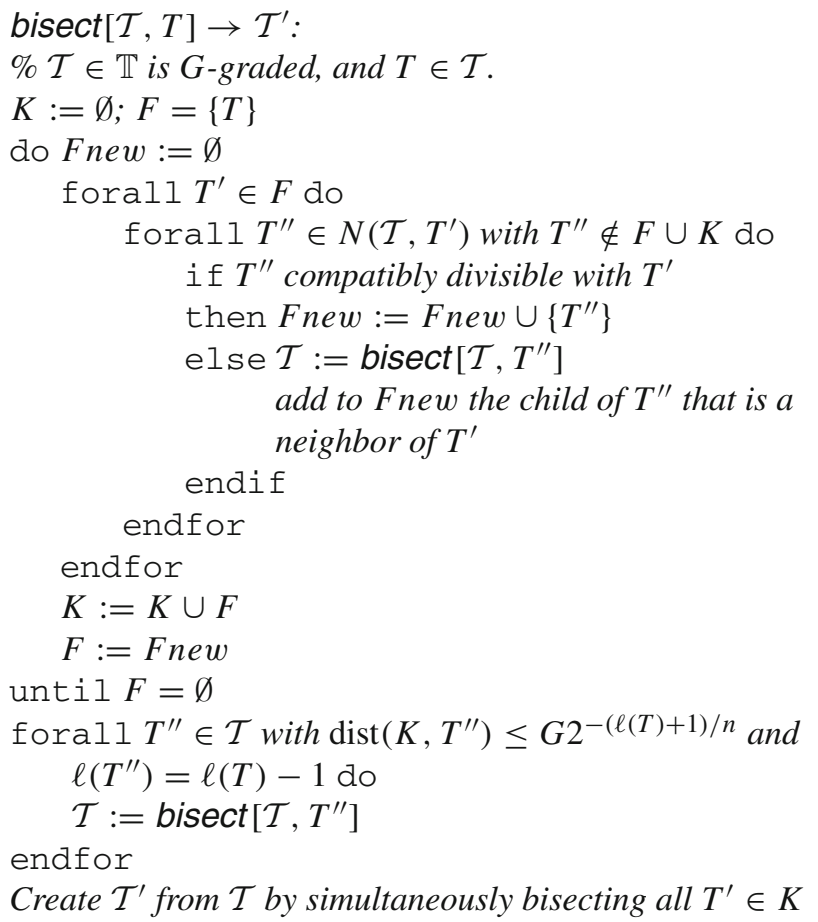

Properties of bisect are stated in the following two lemmas. Similar to [20, Theorem 5.1], the first lemma can be proven by induction to $\ell(T)$.

Proposition $7 \mathcal{T}^{\prime}:=$ bisect $[\mathcal{T}, T]$ terminates, and $\mathcal{T}^{\prime}$ is the smallest G-graded refinement of $\mathcal{T}$ in $\mathbb{T}$ in which $T$ has been bisected.

If $T^{\prime} \in \mathcal{T}^{\prime}$ is newly created by the call, then $\ell\left(T^{\prime}\right) \leq \ell(T)+1$.

Remark 6 Assuming that the data structure allows us to find all neighbors of a simplex in $\mathcal{T} \in \mathbb{T}$ in $\mathcal{O}(1)$ operations, the number of operations needed for $\mathcal{T}^{\prime}:=\operatorname{bisect}[\mathcal{T}, T]$ is $\mathcal{O}\left(\# \mathcal{T}^{\prime}-\# \mathcal{T}\right)$. 
Proposition 8 Any newly created $T^{\prime}$ by the call bisect $[\mathcal{T}, T]$ satisfies

$$
\operatorname{dist}\left(T^{\prime}, T\right) \leq\left(D\left(1+2^{1 / n}\right)+2^{-1 / n} G\right) \sum_{i=\ell\left(T^{\prime}\right)}^{\ell(T)} 2^{-i / n} \quad\left(\approx \operatorname{diam}\left(T^{\prime}\right)\right)
$$

Proof For $\ell(T)=0$, any newly created $T^{\prime}$ is a child of a $\tilde{T}$ that has its refinement edge on $\partial T$, so that $\operatorname{dist}\left(T^{\prime}, T\right)=0$. Note that in this case the sum over $i$ is empty since $\ell\left(T^{\prime}\right)=\ell(T)+1$.

Assuming that the theorem holds for $\ell(T)=\ell-1 \geq 0$, let us consider $T$ with $\ell(T)=\ell$. If $T^{\prime}$ is created by bisection of any simplex from the set $K$, then $\operatorname{dist}\left(T^{\prime}, T\right)=0$ as in the $\ell(T)=0$ case. If $T^{\prime}$ is created by a recursive call bisect $\left[\mathcal{T}, T^{\prime \prime}\right]$, then either $\operatorname{dist}\left(T^{\prime \prime}, T\right)=0$ or

$$
\operatorname{dist}\left(T^{\prime \prime}, T\right) \leq \operatorname{dist}\left(T^{\prime \prime}, K\right)+D 2^{-\ell(T) / n} \leq\left(D+2^{-1 / n} G\right) 2^{-\ell(T) / n},
$$

where we used that for any $\tilde{T} \in K, \ell(\tilde{T})=\ell(T)$ and thus $\operatorname{diam}(\tilde{T}) \leq D 2^{-\ell(T) / n}$. Now by $\ell\left(T^{\prime \prime}\right)=\ell(T)-1$, the induction hypothesis shows that

$$
\begin{aligned}
\operatorname{dist}\left(T^{\prime}, T\right) \leq & \operatorname{dist}\left(T^{\prime}, T^{\prime \prime}\right)+\operatorname{diam}\left(T^{\prime \prime}\right)+\operatorname{dist}\left(T^{\prime \prime}, T\right) \\
\leq & \left(D\left(1+2^{1 / n}\right)+2^{-1 / n} G\right) \sum_{i=\ell\left(T^{\prime}\right)}^{\ell\left(T^{\prime \prime}\right)} 2^{-i / n} \\
& +D 2^{1 / n} 2^{-\ell(T) / n}+\left(D+2^{-1 / n} G\right) 2^{-\ell(T) / n} \\
= & \left(D\left(1+2^{1 / n}\right)+2^{-1 / n} G\right) \sum_{i=\ell\left(T^{\prime}\right)}^{\ell(T)} 2^{-i / n} .
\end{aligned}
$$

Our AFEM is a loop of the following form:

$\mathcal{T}:=\mathcal{T}_{0}$

do mark some set $\mathcal{M} \subset \mathcal{T}$ for bisection

for $T \in \mathcal{M}$ do

if $T \in \mathcal{T} \quad \%$ i.e., if it has not already been bisected as a $\%$ byproduct of a previous call of bisect in this $\%$ for-loop

then $\mathcal{T}:=\operatorname{bisect}[\mathcal{T}, T]$

endif

endfor

until satisfied

Remark 7 Actually, we mark simplices possibly for some fixed number of multiple bisections. By scheduling such multiple bisections as a sequence of single ones, the algorithm can still be written in the above form. 
The following theorem shows the important result that the difference between the cardinalities of the output and initial partition can be bounded by some absolute multiple of the total number of marked simplices. Besides the uniform shape regularity, the proof given in [20] relies only on the result of Proposition 8 and the second statement from Proposition 7. As we have shown, these results are still valid when considering conforming partitions that are additionally $G$-graded, and so is the theorem.

We emphasize that the proof from [20] is a harmless modification of the original, ingenious proof given in [2].

Theorem 4 Let $K$ be the total number of calls of bisect in the above AFEM loop, so that $K$ is no larger than the sum of the cardinalities of all sets of marked simplices. Then for the output partition $\mathcal{T}$, it holds that $\# \mathcal{T}-\# \mathcal{T}_{0} \lesssim K$, dependent only on the constants $d, D, G$, and $n$.

Any descendant $\tilde{\mathcal{T}}$ of $\mathcal{T}_{0}$, not necessarily conforming or $G$-graded, is constructed from $\mathcal{T}_{0}$ by a sequence of bisections, where $\#\left(\tilde{\mathcal{T}} \backslash \mathcal{T}_{0}\right)$ is equal to the number of bisections. By replacing these bisections by calls of bisect, we infer the following consequence of Theorem 4. It shows that there is no essential restriction in considering only $G$-graded partitions from $\mathbb{T}$.

Corollary 5 Any descendant $\tilde{\mathcal{T}}$ of $\mathcal{T}_{0}$ can be refined to a $G$-graded partition $\mathcal{T} \in \mathbb{T}$ with $\# \mathcal{T}-\# \mathcal{T}_{0} \lesssim \# \tilde{\mathcal{T}}-\# \mathcal{T}_{0}$, dependent only on $d, D, G$, and $n$.

Remark 8 For any descendant $\mathcal{T}$ of $\mathcal{T}_{0}$, it holds that $\# \mathcal{T}-\# \mathcal{T}_{0} \leq \#\left(\mathcal{T} \backslash \mathcal{T}_{0}\right) \leq$ $2\left(\# \mathcal{T}-\# \mathcal{T}_{0}\right)$.

\section{A.4 Mesh functions}

In this section we construct piecewise linear mesh functions $h_{\mathcal{T}} \in C(\bar{\Omega}) \cap$ $\prod_{T \in \mathcal{T}} P_{1}(T)$ such that for any $\mu>0,\left\|\nabla h_{\mathcal{T}}\right\|_{L_{\infty}(\Omega)} \leq \mu$ for all $G$-graded $\mathcal{T} \in \mathbb{T}$ with $G \cdot \mu$ sufficiently large, and such that $h_{\left.\mathcal{T}\right|_{T}} \approx h_{T}$ uniformly in $\mu, T \in \mathcal{T}$ and in all those $G$-graded $\mathcal{T} \in \mathbb{T}$. Moreover, these mesh functions will be pointwise non-increasing under conforming and $G$-graded refinements. The class of $G$-graded $\mathcal{T} \in \mathbb{T}$ for this value of $G$ can thus be taken as the class $\mathbb{T}_{\mu}$ as introduced in Sect. 2. In view of Corollary 5 , this class is sufficiently large for our purposes.

For a $\mathcal{T} \in \mathbb{T}, z$ a vertex of $\mathcal{T}$, and $p \in \mathbb{N}_{0}$, we define the patches ("rings")

$$
R_{p}(z, \mathcal{T})= \begin{cases}\cup_{\{T \in \mathcal{T}: T \ni z\}} T & \text { when } p=0 \\ \bigcup_{\left\{z^{\prime}: z^{\prime} \text { is a vertex of } T \in R_{0}(z, \mathcal{T})\right\}} R_{p-1}\left(z^{\prime}, \mathcal{T}\right) & \text { when } p>0\end{cases}
$$

Our approach to constructing a "flat" mesh function $h_{\mathcal{T}}$ is to define $h_{\mathcal{T}}(z)$ as some average of $h_{T}$ over $T \in R_{p}(z, \mathcal{T})$ with $p$ being sufficiently large. To ensure that at the same time it is equivalent to the mesh size near $z$, it will be needed that $G$ is sufficiently large.

In the next proposition, we essentially establish that properly sized element rings in $G$-graded meshes are in fact quasi-uniform, i.e., all elements in such rings are at almost the same refinement level. 
Proposition 9 For $G \geq(2 p+1) D 2^{1 / n}$, a $G$-graded $\mathcal{T} \in \mathbb{T}$, and $z$ a vertex of $\mathcal{T}$, the levels of any two simplices in $R_{p}(z, \mathcal{T})$ differ at most one.

Proof Assume that $T, T^{\prime \prime} \in R_{p}(z, T)$, and in order to reach a contradiction assume in addition that $\ell\left(T^{\prime \prime}\right)<\ell(T)-1$. We take $T^{\prime \prime}$ to be the closest element to $T$ lying in $R_{p}(z, T)$ and satisfying $\ell\left(T^{\prime \prime}\right)<\ell(T)-1$ in the sense that there is a path $P$ along element edges from $T$ to $T^{\prime \prime}$ which lies in $R_{p}(z, T)$, which has the smallest possible number of edges over all such paths from $T$ to $T^{\prime \prime}$, and for which each edge in the path $P$ corresponds to an element $T^{\prime}$ satisfying $\ell\left(T^{\prime}\right) \geq \ell(T)-1$. Since $T, T^{\prime \prime} \in R_{p}(z, T)$, the path $P$ contains at most $2 p+1$ element edges, each of length at most $D 2^{\frac{-\ell(T)+1}{n}}$. Thus $\operatorname{dist}\left(T, T^{\prime \prime}\right) \leq(2 p+1) D 2^{-\frac{\ell(T)+1}{n}} \leq G 2^{-\frac{\ell(T)}{n}}$, which in contradiction to our initial assumption implies that $\ell\left(T^{\prime \prime}\right) \geq \ell(T)-1$ since $\mathcal{T}$ is $G$-graded with $G \geq(2 p+1) D 2^{1 / n}$. Thus $\ell\left(T^{\prime \prime}\right) \geq \ell(T)-1$ and similarly $\ell(T) \geq \ell\left(T^{\prime \prime}\right)-1$. That is, $\ell\left(T^{\prime \prime}\right)+1 \geq \ell(T) \geq \ell\left(T^{\prime \prime}\right)-1$, which is the desired conclusion.

Remark 9 Note that a patch $R_{p}(z, \mathcal{T})$ is not necessarily simply connected. An example is given in Fig. 4.

For $z$ a vertex of a conforming partition $\mathcal{T}$, let

$$
\ell(z):=\min _{\{T \in \mathcal{T}: T \ni z\}} \ell(T)
$$

be the level of $z$ (in $\mathcal{T}$ ). Next it is shown that $|\ell(T)-\ell(z)| \leq 1$ for any $T \in \mathcal{T}$ that intersects an Euclidean ball around $z$ with radius being a fixed multiple of the mesh size near $z$, assuming $G$ be sufficiently large, and thus that the number of such $T$ is uniformly bounded.

Lemma 6 For a $G$-graded $\mathcal{T} \in \mathbb{T}, \rho \in\left(0, G 2^{-2 / n}\right]$, and $z$ a vertex of $\mathcal{T}$, any two vertices in $B\left(z, \rho 2^{-\ell(z) / n}\right) \cap \mathcal{T}$ are connected by a path along the edges of $\mathcal{T}$ of length at most $L$ edges, where $L$ depends only on $\rho, n$ and the shape regularity parameters $d$ and $D$.

Furthermore, for any $T \in \mathcal{T}$ with $T \cap B\left(z, \rho 2^{-\ell(z) / n}\right) \neq \emptyset,|\ell(T)-\ell(z)| \leq 1$.

Proof For all $T \in \mathcal{T}$ with $T \cap B\left(z, \rho 2^{-\ell(z) / n}\right) \neq \emptyset$, it holds that $\ell(T) \leq \ell(z)+1$. Indeed, assume that such a $T$ exists with $\ell(T) \geq \ell(z)+2$. Then, using that the levels of neighboring simplices in $\mathcal{T}$ differ at most one (Corollary $4(1)$ ), there exists

Fig. 4 An example of a non-simply connected $R_{1}(z, \mathcal{T})$

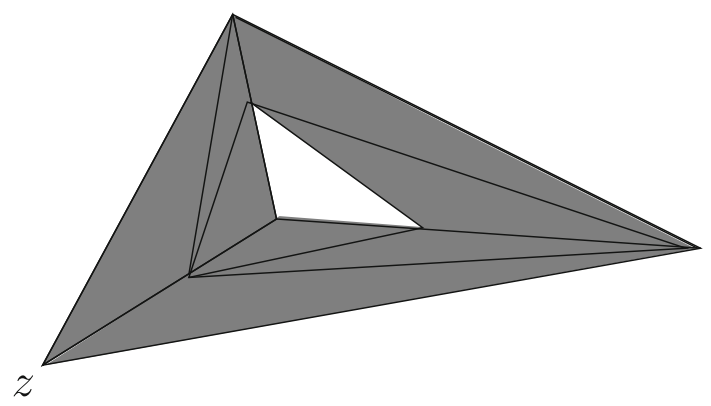


such a $T$ with $\ell(T)=\ell(z)+2$. However, the existence of such a $T$ is in conflict with the $G$-gradedness of the partition since $\operatorname{dist}(T, z) \leq G 2^{-(\ell(z)+2) / n}$. Finally, the $G$-gradedness of the partition also implies that $\ell(T) \geq \ell(z)-1$ for any $T \in \mathcal{T}$ with $T \cap B\left(z, \rho 2^{-\ell(z) / n}\right) \neq \emptyset$.

We infer that the number of $T \in \mathcal{T}$ with $T \cap B\left(z, \rho 2^{-\ell(z) / n}\right) \neq \varnothing$ is uniformly bounded, dependent only on $\rho, n, d$ and $D$, and, consequently, that any two vertices in $B\left(z, \rho 2^{-\ell(z) / n}\right) \cap \mathcal{T}$ are connected by a path along the edges of $\mathcal{T}$ with a length that is uniformly bounded.

In the next proposition it is shown that $\# R_{p}(z, \mathcal{T}) \approx(p+1)^{n}$ when $G \gtrsim p$ (and when $\ell(z)$ is sufficiently large in relation to $p$ since otherwise the ring "overflows" the domain $\Omega$ is all directions). To prove that \# $R_{p}(z, \mathcal{T}) \gtrsim(p+1)^{n}$, it will be demonstrated that if the Euclidean distance between vertices $z, z^{\prime} \in \mathcal{T}$ is $M \lesssim G$ times the local mesh size at $z$ (or equivalently at $z^{\prime}$ since $G$ be sufficiently large), then there exists a path over the edges of $\mathcal{T}$ of length $\mathcal{O}(M)$ that connects $z$ and $z^{\prime}$. This improves upon Lemma 6 where no linear dependence of the path length $L$ on $\rho$ was demonstrated.

Proposition 10 It holds that

$$
\# R_{p}(z, \mathcal{T}) \lesssim(p+1)^{n}
$$

uniformly in $p \in \mathbb{N}_{0}$, all $G$-graded $\mathcal{T} \in \mathbb{T}$ with $G \geq(2 p+1) D 2^{1 / n}$ and vertices $z$ of $\mathcal{T}$, as well as

$$
\# R_{p}(z, \mathcal{T}) \gtrsim(p+1)^{n}
$$

when additionally $p 2^{-\ell(z) / n} \lesssim 1$, dependent only on the domain $\Omega$.

Finally, it holds that

$$
\frac{\# R_{p}(z, \mathcal{T})}{\sum_{q=0}^{p} \# R_{q}(z, \mathcal{T})} \lesssim \frac{1}{p+1}
$$

uniformly in $p \in \mathbb{N}_{0}$, all G-graded partitions $\mathcal{T} \in \mathbb{T}$ with $G \geq(2 p+1) D 2^{1 / n}$ and vertices $z$ of $\mathcal{T}$.

Proof From Proposition 9, we know that $\operatorname{diam}(T) \approx 2^{-\ell(z) / n}$ uniformly in $T \in$ $R_{p}(z, \mathcal{T})$, and thus that $R_{p}(z, \mathcal{T})$ is contained in a ball having radius not larger than some absolute multiple of $(p+1) 2^{-\ell(z) / n}$. Because $\operatorname{vol}(T) \gtrsim 2^{-\ell(z)}$ for $T \in R_{p}(z, \mathcal{T})$, we conclude that $\# R_{p}(z, \mathcal{T}) \lesssim(p+1)^{n}$.

For any $z \in \mathcal{T}$, there exists a cone $C$ with vertex $z$ and fixed height and opening, dependent only on $\Omega$, such that $C \subset \bar{\Omega}$. We are going to show that there exists a constant $c_{\text {st }}>0$, such that for all $M \geq 1$,

$$
C \cap B\left(z, M 2^{-\ell(z) / n}\right) \cap \mathcal{T} \subset R_{p}(z, \mathcal{T}) \quad\left(G \geq(2 p+1) D 2^{1 / n}, p \geq c_{\mathrm{st}} M\right) .
$$

Since $\operatorname{vol}\left(B\left(z, M 2^{-\ell(z) / n}\right) \cap C\right) \gtrsim M^{n} 2^{-\ell(z)}$ when $M 2^{-\ell(z) / n} \lesssim 1$, dependent only on the sizes of $C$, and $\operatorname{vol}(T) \lesssim 2^{-\ell(z)}$ for $T \in R_{p}(z, \mathcal{T}),(8.1)$ implies that $\# R_{p}(z, \mathcal{T}) \gtrsim$ $(p+1)^{n}$ when $p 2^{-\ell(z) / n} \lesssim 1$. 
Let $\rho$ be a fixed, sufficiently large constant to be determined below. Since $G \geq(2 p+$ 1) $D 2^{1 / n}$ and $p \geq c_{\mathrm{st}} M \geq c_{\mathrm{st}}$, by taking $c_{\text {st }}$ sufficiently large, we have $\rho<G 2^{-2 / n}$.

Given $M \geq 1$, let $z^{\prime} \in C \cap B\left(z, M 2^{-\ell(z) / n}\right)$ be a vertex of $\mathcal{T}$ and $M^{\prime}:=\mid z-$ $z^{\prime} \mid 2^{\ell(z) / n}$. If $\bar{M}^{\prime} \leq \rho$, i.e., $z^{\prime} \in B\left(z, \rho 2^{-\ell(z) / n}\right)$, then, by taking $c_{\text {st }} \geq L-1$, where $L=L(\rho, n, d, D) \in \mathbb{N}$ is the constant from Lemma 6 , we have $p \geq c_{\mathrm{st}} M \geq L-1$, and so $z^{\prime}$ is a vertex in $R_{p}(z, \mathcal{T})$ thanks to this lemma.

Now let $M^{\prime}>\rho$. Since for any $T \in \mathcal{T}$ with $T \cap B\left(z, \rho 2^{-\ell(z) / n}\right) \neq \emptyset, \mid \ell(T)-$ $\ell(z) \mid \leq 1$ by Lemma 6 , for $\rho$ being sufficiently large in relation to the opening angle $\alpha$ of the cone $C$ and the shape regularity parameter $D$, there exists a vertex $z_{1}$ of $\mathcal{T}$ in $C \cap B\left(z, \rho 2^{-\ell(z) / n}\right)$ with

$$
\left|z_{1}-z^{\prime}\right| \leq\left(M^{\prime}-\frac{1}{2} \rho\right) 2^{-\ell(z) / n}
$$

See Fig. 5 for an illustration.

With $z_{0}:=z$, a repeated application of this argument shows that there exists a sequence of vertices $z_{1}, z_{2}, \ldots$ of $\mathcal{T}$ with $z_{i}$ in $C \cap B\left(z_{i-1}, \rho 2^{-\ell\left(z_{i-1}\right) / n}\right)$ and

$$
\left|z_{i}-z^{\prime}\right| \leq\left|z_{i-1}-z^{\prime}\right|-\frac{1}{2} \rho 2^{-\ell\left(z_{i-1}\right) / n}
$$

whenever $\left|z_{i-1}-z^{\prime}\right|>\rho 2^{-\ell\left(z_{i-1}\right) / n}$, and with $z_{i}=z^{\prime}$ otherwise.

Let $m \geq 2$ be the smallest integer with $M-\frac{1}{2} \rho-(m-1) \frac{1}{2} 2^{-\frac{1}{n}} \rho \leq 0$. When $p \geq(m-1) L$, for $1 \leq i \leq m-1$ we have that any $T \in \mathcal{T}$ with $T \ni z_{i}$ is in $R_{p}(z, \mathcal{T})$ by Lemma 6 . Taking $G \geq(2 p+1) D 2^{1 / n}$ then ensures that $\left|\ell\left(z_{i}\right)-\ell(z)\right| \leq 1$ by Proposition 9. By substituting $\ell\left(z_{i-1}\right) \leq \ell(z)+1$ in (8.2), we conclude that $z_{m}=z^{\prime}$. In addition requiring that $p \geq m L-1$ implies that $z^{\prime}=z_{m} \in R_{p}(z, \mathcal{T})$.

Since $m \lesssim M$, our assumption that $p \geq m L-1$ is satisfied when $c_{\text {st }}$ is sufficiently large, with which we have completed the proof of (8.1), and thus that of $\# R_{p}(z, \mathcal{T}) \gtrsim(p+1)^{n}$ whenever $p 2^{-\ell(z) / n} \lesssim 1$.

Combining the upper and lower bounds for $\# R_{p}(z, \mathcal{T})$ yields the last statement of this Proposition in the case when $p 2^{-\ell(z) / n} \lesssim 1$. The above arguments show that the lower bound $\# R_{p}(z, \mathcal{T}) \gtrsim(p+1)^{n}$ will only be violated when $p$ is large enough that

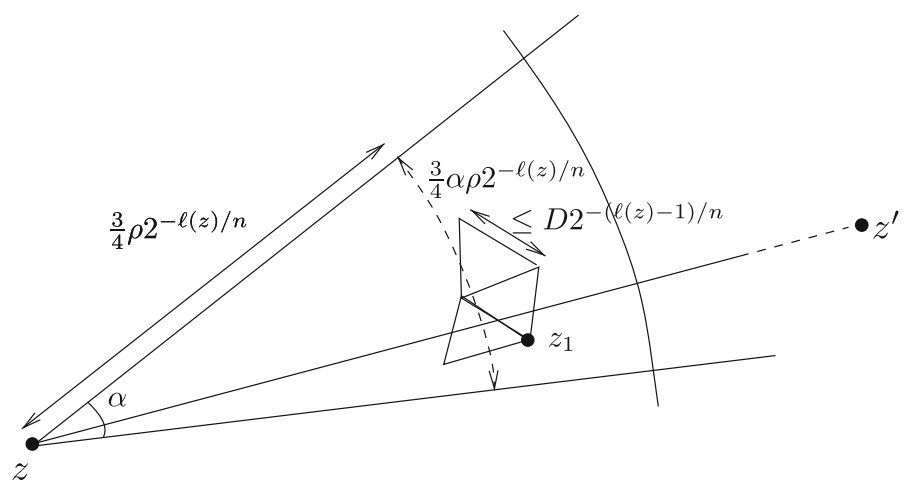

Fig. 5 Illustration with the proof of Proposition 10 
$R_{p}(z, \mathcal{T})$ (nearly) fills the domain, i.e., that it is equal to $\mathcal{T}$. From that value of $p$ on, $\# R_{p}(z, \mathcal{T})$ will not grow anymore, from which we infer the last statement also in that case.

For any $\mathcal{T} \in \mathbb{T}$, we define $\bar{h}_{\mathcal{T}, p} \in C(\bar{\Omega}) \cap \prod_{T \in \mathcal{T}} P_{1}(T)$ by

$$
\bar{h}_{\mathcal{T}, p}(z)=\frac{\sum_{q=0}^{p} \sum_{T \in R_{q}(z, \mathcal{T})} h_{T}}{\sum_{q=0}^{p} \# R_{q}(z, \mathcal{T})}
$$

for any vertex $z$ of $\mathcal{T}$. The next proposition shows that $\bar{h}_{\mathcal{T}, p}$ is appropriately called a mesh function. The proof follows from Proposition 9 and the uniform shape regularity.

Proposition 11 For $G$-graded $\mathcal{T} \in \mathbb{T}$, it holds that

$$
\left.\bar{h}_{\mathcal{T}, p}\right|_{T} \approx h_{T}
$$

uniformly in $p \in \mathbb{N}_{0}, G \geq(2 p+1) D 2^{1 / n}$, and $T \in \mathcal{T}$.

Next we show that $\bar{h}_{\mathcal{T}, p}$ can be made arbitrarily flat by decreasing the grading of the partition, i.e., by increasing $G$, and by increasing $p$ proportionally.

Proposition 12 For $G$-graded $\mathcal{T} \in \mathbb{T}$, it holds that

$$
\left|\bar{h}_{\mathcal{T}, p}(z)-\bar{h}_{\mathcal{T}, p}\left(z^{\prime}\right)\right| \lesssim \frac{1}{p+1} h_{T}
$$

uniformly in $p \in \mathbb{N}_{0}, G \geq(2 p+1) D 2^{1 / n}, T \in \mathcal{T}$, and vertices $z, z^{\prime} \in T$, and so $\left\|\nabla \bar{h}_{\mathcal{T}, p}\right\|_{L_{\infty}(\Omega)} \lesssim \frac{1}{p+1}$

Proof For $z^{\prime \prime} \in\left\{z, z^{\prime}\right\}$, let

$$
n\left(z^{\prime \prime}\right)=\sum_{q=0}^{p} \sum_{T^{\prime} \in R_{q}\left(z^{\prime \prime}, \mathcal{T}\right)} h_{T^{\prime}}, \quad d\left(z^{\prime \prime}\right)=\sum_{q=0}^{p} \# R_{q}\left(z^{\prime \prime}, \mathcal{T}\right) .
$$

Letting $R_{-1}(\cdot, \cdot):=\emptyset$, we have $R_{q}\left(z^{\prime \prime}, \mathcal{T}\right) \backslash\left(R_{q}(z, \mathcal{T}) \cap R_{q}\left(z^{\prime}, \mathcal{T}\right)\right) \subset R_{q}\left(z^{\prime \prime}, \mathcal{T}\right) \backslash$ $R_{q-1}\left(z^{\prime \prime}, \mathcal{T}\right)$. Employing Proposition 10 , we compute

$$
\sum_{q=0}^{p} \#\left(R_{q}\left(z^{\prime \prime}, \mathcal{T}\right) \backslash R_{q-1}\left(z^{\prime \prime}, \mathcal{T}\right)\right)=\# R_{p}\left(z^{\prime \prime}, \mathcal{T}\right) \lesssim \frac{1}{p+1} d\left(z^{\prime \prime}\right)
$$

Applying Proposition 9 then yields $\left|n(z)-n\left(z^{\prime}\right)\right| \lesssim \frac{h_{T}}{p+1}\left(d(z)+d\left(z^{\prime}\right)\right),\left|d(z)-d\left(z^{\prime}\right)\right| \lesssim$ $\frac{1}{p+1}\left(d(z)+d\left(z^{\prime}\right)\right)$, and $\left|\frac{n\left(z^{\prime \prime}\right)}{d\left(z^{\prime \prime}\right)}\right| \lesssim h_{T}$. Assuming $d\left(z^{\prime}\right) \geq d(z)$, we may complete the proof by writing 


$$
\frac{n(z)}{d(z)}-\frac{n\left(z^{\prime}\right)}{d\left(z^{\prime}\right)}=\frac{n(z)}{d(z)} \frac{d\left(z^{\prime}\right)-d(z)}{d\left(z^{\prime}\right)}+\frac{n(z)-n\left(z^{\prime}\right)}{d\left(z^{\prime}\right)}
$$

Remark 10 Proposition 11 is still valid if the more obvious definition $\bar{h}_{\mathcal{T}, p}=$ $\sum_{T \in R_{p}(z, \mathcal{T})} h_{T} / \# R_{p}(z, \mathcal{T})$ of the mesh function is used. In order to prove a result such as Proposition 12 for this mesh function, however, it is necessary to show that, for $G \geq(2 p+1) D 2^{1 / n}, \#\left(R_{p}\left(z^{\prime \prime}, \mathcal{T}\right) \backslash R_{p-1}\left(z^{\prime \prime}, \mathcal{T}\right)\right) / \# R_{p}\left(z^{\prime \prime}, \mathcal{T}\right) \lesssim \frac{1}{p+1}$, or at least that it tends to zero for $p \rightarrow \infty$. It turns out that such an estimate is difficult to establish, cf. also Remark 9.

For some applications it is important to have a family of mesh functions that is non-increasing under (conforming, $G$-graded) refinements. For $G$-graded $\mathcal{T} \in \mathbb{T}$, we define $h_{\mathcal{T}, p} \in C(\bar{\Omega}) \cap \prod_{T \in \mathcal{T}} P_{1}(T)$ by

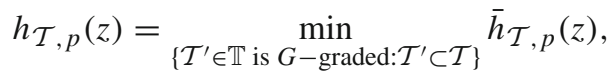

where $z$ is any vertex of $\mathcal{T}$.

Proposition 13 The mapping $\mathbb{T} \ni \mathcal{T} \mapsto h_{\mathcal{T}, p}$ is pointwise non-increasing under $G$ graded refinements, and for some constants $c_{\mathbb{T}}, C_{\mathbb{T}}, \tilde{C}_{\mathbb{T}}$, which are independent of $p$, it holds that

$$
c_{\mathbb{T}} h_{T} \leq\left. h_{\mathcal{T}, p}\right|_{T} \leq C_{\mathbb{T}} h_{T}, \quad\left\|\nabla h_{\mathcal{T}, p}\right\|_{L_{\infty}(\Omega)} \leq \tilde{C}_{\mathbb{T}} \frac{1}{p+1},
$$

for all $G$-graded $\mathcal{T} \in \mathbb{T}$ with $G \geq(2 p+1) D 2^{1 / n}$, and $T \in \mathcal{T}$.

Proof The first two statements follow by definition of $h_{\mathcal{T}, p}$ and Proposition 11.

For vertices $z_{1}, z_{2}$ of $\mathcal{T}$, let $\bar{h}_{\mathcal{T}_{1}, p}\left(z_{1}\right)=h_{\mathcal{T}, p}\left(z_{1}\right) \leq h_{\mathcal{T}, p}\left(z_{2}\right)=\bar{h}_{\mathcal{T}_{2}, p}\left(z_{2}\right)$. Then we have $\left|h_{\mathcal{T}, p}\left(z_{1}\right)-h_{\mathcal{T}, p}\left(z_{2}\right)\right| \leq\left|\bar{h}_{\mathcal{T}_{1}, p}\left(z_{1}\right)-\bar{h}_{\mathcal{T}_{1}, p}\left(z_{2}\right)\right| \leq\left\|\nabla \bar{h}_{\mathcal{T}_{1}}\right\|_{L_{\infty}(\Omega)}\left\|z_{1}-z_{2}\right\|$. Now an application of Proposition 12 shows the second statement.

We conclude that given a $\mu>0$, for $p+1 \geq \tilde{C}_{\mathbb{T}} \mu^{-1}$ and $G \geq(2 p+1) D 2^{1 / n}$, the collection of all $G$-graded $\mathcal{T} \in \mathbb{T}$ can be used as the class $\mathbb{T}_{\mu}$.

\section{References}

1. Babuška, I., Osborn, J.: Analysis of finite element methods for second order boundary value problems using mesh dependent norms. Numer. Math. 34, 41-62 (1980)

2. Binev, P., Dahmen, W., DeVore, R.: Adaptive finite element methods with convergence rates. Numer. Math. 97, 219-268 (2004)

3. Brenner, S.C., Scott, L.R.: The Mathematical Theory of Finite Element Methods. Texts in Applied Mathematics, vol. 15, 3rd edn. Springer, New York (2008)

4. Cascon, J., Kreuzer, C., Nochetto, R.H., Siebert, K.G.: Quasi-optimal convergence rate for an adaptive finite element method. SIAM J. Numer. Anal. 46, 2524-2550 (2008)

5. Demlow, A.: Convergence of an adaptive finite element method for controlling local energy errors. SIAM J. Numer. Anal. 48(2), 470-497 (2010) 
6. Dörfler, W.: A convergent adaptive algorithm for Poisson's equation. SIAM J. Numer. Anal. 33, 1106-1124 (1996)

7. Eriksson, K.: An adaptive finite element method with efficient maximum norm error control for elliptic problems. Math. Models Methods Appl. Sci. 4, 313-329 (1994)

8. Eriksson, K., Johnson, C.: Adaptive finite element methods for parabolic problems. II. Optimal error estimates in $L_{\infty} L_{2}$ and $L_{\infty} L_{\infty}$. SIAM J. Numer. Anal. 32, 706-740 (1995)

9. Liao, X., Nochetto, R.H.: Local a posteriori error estimates and adaptive control of pollution effects. Numer. Methods Partial Differ. Equ. 19, 421-442 (2003)

10. Maubach, J.: Local bisection refinement for $n$-simplicial grids generated by reflection. SIAM J. Sci. Comput. 16, 210-227 (1995)

11. Mekchay, K., Nochetto, R.H.: Convergence of adaptive finite element methods for general second order linear elliptic PDEs. SIAM J. Numer. Anal. 43, 1803-1827 (2005) (electronic)

12. Morin, P., Nochetto, R.H., Siebert, K.G.: Convergence of adaptive finite element methods. SIAM Rev. 44, 631-658 (2002) (electronic) (2003) Revised reprint of "Data oscillation and convergence of adaptive FEM" [SIAM J. Numer. Anal. 38(2), 466-488 (2000) (electronic); MR1770058 (2001g:65157)]

13. Morin, P., Siebert, K., Veeser, A.: Convergence of Finite Elements Adapted for Weaker Norms. Applied and Industrial Mathematics in Italy II. Ser. Adv. Math. Appl. Sci., vol. 75, pp. 468-479. World Sci. Publ., Hackensack, NJ (2007)

14. Morin, P., Siebert, K., Veeser, A.: A basic convergence result for conforming adaptive finite elements. Math. Models Methods Appl. Sci. 18, 707-737 (2008)

15. Nitsche, J.A., Schatz, A.H.: Interior estimates for Ritz-Galerkin methods. Math. Comput. 28, 937-958 (1974)

16. Nochetto, R., Siebert, K., Veeser, A.: Theory of adaptive finite element methods: an introduction. In: DeVore, R., Kunoth, A. (eds.) Multiscale, Nonlinear and Adaptive Approximation: Dedicated to Wolfgang Dahmen on the Occasion of his 60th Birthday, pp. 409-542. Springer, Berlin (2009)

17. Schmidt, A., Siebert, K.G.: Design of adaptive finite element software. The finite element toolbox ALBERTA, with 1 CD-ROM (Unix/Linux). Lecture Notes in Computational Science and Engineering, vol. 42, pp. xii+315. Springer, Berlin (2005)

18. Scott, L.R., Zhang, S.: Finite element interpolation of nonsmooth functions satisfying boundary conditions. Math. Comput. 54, 483-493 (1990)

19. Stevenson, R.: Optimality of a standard adaptive finite element method. Found. Comput. Math. 7, 245-269 (2007)

20. Stevenson, R.: The completion of locally refined simplicial partitions created by bisection. Math. Comput. 77, 227-241 (2008)

21. Traxler, C.T.: An algorithm for adaptive mesh refinement in $n$ dimensions. Computing 59, 115-137 (1997)

22. Verfürth, R.: A Review of a Posteriori Error Estimation and Adaptive Mesh-Refinement Techniques. Wiley-Teubner, Chichester (1996)

23. Wihler, T.P.: Weighted $L^{2}$-norm a posteriori error estimation of FEM in polygons. Int. J. Numer. Anal. Model. 4, 100-115 (2007)

24. Xu, J., Zhou, A.: Local and parallel finite element algorithms based on two-grid discretizations. Math. Comput. 69, 881-909 (2000) 